\title{
Interferon- $\beta$ induced in female genital epithelium by HIV-1 glycoprotein 120 via Toll-like-receptor 2 pathway acts to protect the mucosal barrier
}

\author{
Aisha Nazli ${ }^{1,2}$, Sara Dizzell ${ }^{1,2}$, Muhammad Atif Zahoor ${ }^{1,2}$, Victor H Ferreira ${ }^{1,2}$, Jessica Kafka ${ }^{1,2}$, \\ Matthew William Woods ${ }^{1,2}$, Michel Ouellet ${ }^{3}$, Ali A Ashkar ${ }^{1,2}$, Michel J Tremblay ${ }^{3}$, \\ Dawn ME Bowdish ${ }^{1,2}$ and Charu Kaushic ${ }^{1,2}$
}

More than $40 \%$ of HIV infections occur via female reproductive tract (FRT) through heterosexual transmission. Epithelial cells that line the female genital mucosa are the first line of defense against HIV-1 and other sexually transmitted pathogens. These sentient cells recognize and respond to external stimuli by induction of a range of carefully balanced innate immune responses. Previously, we have shown that in response to HIV-1 gp120, the genital epithelial cells (GECs) from upper reproductive tract induce an inflammatory response that may facilitate HIV-1 translocation and infection. In this study, we report that the endometrial and endocervical GECs

simultaneously induce biologically active interferon- $\beta$ (IFN $\beta$ ) antiviral responses following exposure to HIV- 1 that act to protect the epithelial tight junction barrier. The innate antiviral response was directly induced by HIV-1 envelope glycoprotein gp120 and addition of gp120 neutralizing antibody inhibited IFN $\beta$ production. Interferon- $\beta$ was induced by gp120 in upper GECs through Toll-like receptor 2 signaling and required presence of heparan sulfate on epithelial cell surface. The induction of IFN $\beta$ was dependent upon activation of transcription factor IRF3 (interferon regulatory factor 3 ). The IFN $\beta$ was biologically active, had a protective effect on epithelial tight junction barrier and was able to inhibit HIV-1 infection in TZM-bl indicator cells and HIV-1 replication in T cells. This is the first report that recognition of HIV-1 by upper GECs leads to induction of innate antiviral pathways. This could explain the overall low infectivity of HIV-1 in the FRT and could be exploited for HIV-1 prophylaxis. Cellular and Molecular Immunology advance online publication, 19 March 2018; doi:10.1038/cmi.2017.168

Keywords: antiviral response; genital epithelium; HIV-1; IFN- $\beta$; innate immunity; IRF-3; mucosal barrier; TLR2

\section{INTRODUCTION}

Almost 70\% of HIV infection occurs via sexual transmission in the intestinal or genital tract. Globally, women constitute more than half of 36.9 million HIV-infected individuals. HIV and AIDS are the leading causes of death worldwide among women of reproductive age. Clinical and experimental studies indicate that HIV can be transmitted through both the upper (oviduct, uterus and endocervix) and lower (ectocervix, vagina) genital tract. In particular, the transformation zone in the cervix is considered to be a highly susceptible site because of the presence of a large number of target cells in the lamina propria, below the epithelium. ${ }^{1}$ However, despite the relatively large surface area available for HIV-1 exposure and the higher incidence in women, the overall transmission in female reproductive tract (FRT) is relatively inefficient, estimated at 1:200 to 1:2000 per coital act, indicating that the FRT provides a significant barrier to HIV transmission. ${ }^{2}$

Following HIV-1 exposure, the acute events that determine the outcome of the interaction with the virus in the FRT are still not well understood. HIV needs to cross the epithelial lining of the genital mucosa in order to establish infection in the female genital tract. There is general consensus that the

\footnotetext{
${ }^{1}$ McMaster Immunology Research Centre, Michael G. DeGroote Center for Learning and Discovery, McMaster University, Hamilton, ON L8S 4K1, Canada; ${ }^{2}$ Department of Pathology and Molecular Medicine, McMaster University, Hamilton, ON L8S 4K1, Canada and ${ }^{3}$ Department of Medical Biology, Laval University, Quebec City, QC G1V OA6, Canada

Correspondence: Dr C Kaushic, PhD, Department of Pathology and Molecular Medicine, McMaster University, 1280 Main Street West, MDCL 4014 , Hamilton, ON L8S 4K1, Canada.

E-mail: kaushic@mcmaster.ca

Received: 7 September 2017; Revised: 12 December 2017; Accepted: 12 December 2017
} 
epithelial cells themselves do not get infected. ${ }^{1}$ However, there is increasing evidence that they play a key role as sensors and first responders for the host innate immune system, in addition to forming a physical and functional barrier against microorganisms. ${ }^{3}$

The upper genital epithelial cells (GECs) are dynamically active cells that display a carefully orchestrated response to external stimuli. They perform a multitude of tasks in encounters with pathogens, including induction of innate responses, as well as relaying signals to activate other cells of the innate and adaptive immune system. Both the upper and lower genital tract epithelium have been shown to express antimicrobial peptides as well as a repertoire of pathogen pattern recognition receptors like Toll-like receptors (TLRs) that allows them to respond to a wide array of pathogens and initiate innate and adaptive responses. ${ }^{4}$ We and others have reported the induction of innate antiviral responses in upper GEC cultures following treatment with TLR ligands, resulting in decreased HSV-2 replication. The TLR3 ligand polyinosinic: polycytidylic acid (poly I:C) was shown to induce the greatest antiviral effect, but this was accompanied with enhanced production of inflammatory cytokines. ${ }^{5,6}$ The antiviral effect by upper GECs was mediated by production of interferon- $\beta$ (IFN $\beta$ ) in response to TLR ligands. Other studies have also reported production of interferon-stimulated factors such as MyxA, 2' $5^{\prime}$-oligoadenylate synthetase (OAS) and inducible nitric oxide synthase that have direct antiviral effects in primary cultures of endometrial epithelial cells as well as cervical and cervicovaginal cell lines. ${ }^{6,7}$

Type I interferons (IFNs) play a mixed role in HIV-1 infection. In general, production of IFN in the acute phase is associated with protection against infection and control of viral replication, although it also leads to immune activation and influx of other immune cells that supports viral replication. ${ }^{8,9}$ The protective role played by type I IFN is associated with inhibition of HIV-1 replication because of upregulation of a number of IFN-stimulated genes (ISGs). ${ }^{10-14}$ Plasmacytoid dendritic cells ( $\mathrm{pDCs}$ ) in particular have been reported to be a rich source of IFN during HIV-1 infection and studies in elite controllers, HIV-1-infected individuals who maintain undetectable viral load in the absence of antiretroviral therapy, show that pDCs counts correlate with IFN production. . $^{15,16}$

Previously, we have reported that the upper GECs do not get productively infected with HIV-1. ${ }^{17}$ Primary epithelial cells isolated from endometrium and cervix responded directly to HIV-1, resulting in production of inflammatory cytokines that disrupted the tight junctions forming the epithelial barrier, allowing translocation of the virus and luminal bacteria. ${ }^{18}$ Further studies revealed that pattern recognition receptors TLR2 and TLR4 present on the cell surface recognized HIV-1 envelope glycoprotein gp120 that activated nuclear factor (NF)- $\mathrm{\kappa B}$ pathway and led to production of proinflammatory cytokine production. ${ }^{19}$ However, no studies have examined whether HIV-1 exposure can activate innate antiviral immunity in the mucosal epithelium of the genital tract and whether this could play a role in inhibiting HIV-1 infection and replication.

In the current study we analyzed the antiviral responses of the genital epithelium from upper reproductive tract and determined the role it may play in preventing HIV infection and replication.

\section{MATERIALS AND METHODS}

Cell lines, reagents and antibodies

Vero cell line (ATCC, Manassas, VA, USA) was used for analyzing biological activity of type I IFN. Vero cells were grown in $\alpha$-minimum essential medium ( $\alpha$-MEM; Invitrogen, Burlington, ON, Canada), supplemented with $10 \%$ heatinactivated fetal bovine serum (FBS; Invitrogen, Burlington, ON, Canada). The human embryonic kidney-293 (HEK293) cell line (ATCC) was used for transfection assays. Cells were grown in Dulbecco's modified Eagle's medium (DMEM; Invitrogen, Burlington, ON, Canada) supplemented with $10 \%$ FBS (Invitrogen). The following reagents were used for the study: tri-palmitoylated lipopeptide Pam3CSK (InvivoGen, San Diego, CA, USA), lipopolysaccharide (LPS) from Escherichia coli 026:B6 (Invitrogen; TLR4 ligand), the novel TLR4 ligand FimH, poly I:C (TLR3 ligand; Sigma Aldrich, Burlington, ON, Canada), BX795 (InvivoGen), heparinase III from Flavobacterium heparinum (Sigma Aldrich) and heparan sulfate (Sigma Aldrich). Recombinant HIV-1 gp120 protein (strain ADA and Bal) was obtained through NIH AIDS Research and Reference Reagent program, Division of AIDS, National Institute of Allergy and Infectious Diseases (NIAID) (Bethesda, MD, USA), National Institutes of Health (NIH) (Bethesda, MD, USA), USA. Subsequently, gp120 was bought from a commercial source (Immunodiagnostic Inc., Woburn, MA, USA). Recombinant gp120 from both sources was compared and commercial source gp120 was used exclusively after obtaining comparable results. The following antibodies were used: anti-human CD282/TLR2, clone TL2.1 (eBioscience, San Diego, CA, USA), mouse IgG2a K isotype control antibody (eBioscience), anti-human CD284/TLR4, clone HTA125 (eBioscience), anti-human CD285/TLR5 (InvivoGen), antigp120 neutralizing antibody, clone 2G12 (Polymun Scientific, Klosterneuburg, Austria), mouse anti-IRF3 (Santa Cruz Biotechnology, Inc., Santa Cruz, CA, USA) and rabbit anti-NF- $\kappa B$ p65 (Santa Cruz Biotechnology, Inc.), anti-IFN $\beta$ antibody $(25 \mu \mathrm{g} / \mathrm{ml}$; Merck Millipore, Etobicoke, ON, Canada) and anti-TNF $\alpha$ antibody $(25 \mu \mathrm{g} / \mathrm{ml}$; Rockland Immunochemicals Inc., Limerick, PA, USA).

\section{Ethics statement}

Primary genital epithelial cells were isolated from cervical and endometrial tissues obtained from women aged 30-59 years

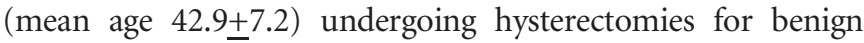
gynecological reasons at Hamilton Health Sciences Hospital. Informed written consent was obtained in accordance with the approval of the Hamilton Integrated Human Research Ethics Board. 


\section{Primary endometrial and cervical epithelial cell cultures}

All experiments were conducted on primary epithelial cell cultures isolated from hysterectomy tissues. Detailed protocol for isolation and culture of primary endometrial and endocervical epithelial cultures, referred to as GECs hereon, has been described previously. ${ }^{20,21}$ Briefly, endometrial and endocervical tissues were separately minced into small pieces and digested in an enzyme mixture at $37^{\circ} \mathrm{C}$. The GECs were isolated by a series of separations through nylon mesh filters (Small Parts Inc., Miramar, FL, USA), resuspended in DMEM/ F12 primary growth medium (Invitrogen) and seeded onto Matrigel $^{\mathrm{TM}}$ (Becton Dickinson and Company, Mississauga, ON, Canada)-coated tissue culture inserts (BD Falcon, Mississauga, ON, Canada). Epithelial cultures were grown for 57 days until confluent monolayers were formed. The confluency was monitored by transepithelial resistance (TER) measured by a volt ohm meter (EVOM; World Precision Instruments, Sarasota, FL, USA). Confluent monolayers showing TER values greater than $1000 \Omega / \mathrm{cm}$ were used for further experiments. The purity of epithelial monolayers was between 95 and 98\%, with no trace of any hematopoietic cells. The detailed protocol for measuring purity of upper genital epithelial cultures has been previously described. ${ }^{20}$

\section{Viral strains and GEC exposure}

HIV-1 ADA (M-tropic) was prepared by infection of primary adherent monocyte-derived macrophages from human peripheral blood mononuclear cells, followed by virus concentration using the Amicon Ultra-15 filtration system (Millipore, Billerica, MA, USA). Env-deleted HIV-1 (env ${ }^{-}$) was kindly provided by $\mathrm{Dr}$ D. Johnson (NCI, NIH, USA), and was prepared on an NL4-3 backbone. For viral exposure, primary GECs were grown to confluency on transwell inserts and subsequently exposed to $100 \mu \mathrm{l}$ of HIV-ADA $\left(10^{6}\right.$ infectious viral unit (IU)/ml, corresponding to a multiplicity of infection (MOI) of 1), ultraviolet (UV)-inactivated HIV (10 $\mathrm{IU} / \mathrm{ml}), \mathrm{HIV}^{6} 1 \mathrm{env}^{-}$(at a p24 concentration of $79 \mathrm{ng} / \mathrm{ml})$ or recombinant gp120 (100 ng/ml) for various time points, depending on the experiment.

To explore the role of TLR on antiviral signaling in GECs by HIV-1, confluent primary cells were treated with neutralizing antibodies against TLR2, 4, 5, isotype antibody (all at $10 \mu \mathrm{g} / \mathrm{ml}$ ) and heparan sulfate $(40 \mu \mathrm{g} / \mathrm{ml})$ during the course of gp120 exposure or pretreatment with heparinase III $(6 \mathrm{mIU} / \mathrm{ml})$ for $1 \mathrm{~h}$ before gp120 exposure. The interferon regulatory factor 3 (IRF3)-mediated pathways were also investigated by pretreating cells with BX795 (an inhibitor of TBK-1/IKKe phosphorylation, an upstream event in activation of IRF3) at a concentration of $1 \mu \mathrm{M}$ for $1 \mathrm{~h}$ before experimental exposure of HIV-1 or gp120. To determine the role of NF- $\kappa B$ in the induction of IFN $\beta$, GEC monolayers were pretreated with the NF- $\kappa B$ inhibitor pyrrolidine dithiocarbamate $(10 \mu \mathrm{M}$, Sigma-Aldrich) for $1 \mathrm{~h}$ before exposure with gp120.

\section{HIV-1 infection assay in TZM-bl cell line}

The TZM-bl indicator cell line (obtained through NIH AIDS Research and Reference Reagent Program, Division of AIDS,
NIAID, NIH: TZM-bl from Dr John C Kappes, Dr Xiaoyun Wu and Tranzyme Inc. (Research Triangle Park, NC, USA) $)^{22-25}$ was maintained in DMEM supplemented with $10 \%$ heat-inactivated FBS (Invitrogen). The cell line was used for quantitative analysis of HIV infection using luciferase as a reporter.

Briefly, 60000 TZM-bl cells were seeded into a 24-well plate in $500 \mu \mathrm{l}$ of $10 \%$ DMEM. Various treatments containing either media alone or recombinant IFN $\beta(100 \mathrm{pg} / \mathrm{ml})$ or GEC supernatants from mock control or gp120-treated monolayers incubated in the presence or absence of rabbit anti-human IFN $\beta$ (AB1431; Millipore, Etobicoke, ON, Canada) or rabbit isotype IgG control (ab172730; Abcam Inc., Toronto, ON, Canada) at $10 \mu \mathrm{g} / \mathrm{ml}$ concentrations were added to TZM-bl cells in a total volume of $250 \mu \mathrm{l}$. Each sample was added to TZM-bl cells in the presence of HIV-1 for $2 \mathrm{~h}$ with a gentle shaking every $15 \mathrm{~min}$. After $2 \mathrm{~h}, 1 \mathrm{ml}$ of $10 \%$ DMEM was overlaid and the cells were further incubated for $24 \mathrm{~h}$ at $37^{\circ} \mathrm{C}$. After $24 \mathrm{~h}$, the cells were washed once with $1 \times$ phosphatebuffered saline, lysed and the luciferase activity was measured using a Stratagene luciferase assay kit (Agilent Technologies, Mississauga, ON, Canada) as per the manufacturer's instructions.

\section{Type 1 IFN bioassay}

To assess the presence of biologically active IFN, a vesicular stomatitis virus (VSV) plaque reduction assay was used. This method is based on assaying the ability of VSV-GFP, a lytic but IFN-sensitive virus that expresses green fluorescent protein (GFP) under the control of a virus promoter (kindly provided by Dr B. Lichty, McMaster University), to replicate within cultures. This assay has been used previously to assess the presence of biologically active type I IFN. Briefly, the supernatants were collected from primary epithelial cells after treatments and diluted with $\alpha$-MEM, and added to plates with Vero cells grown to $80 \%$ confluency. After $24 \mathrm{~h}$, samples were removed, and Vero cells were challenged with VSV-GFP. After $1 \mathrm{~h}$, the unattached VSV-GFP was removed and Vero cells were overlaid with $2 \%$ methylcellulose/ $2 \times$ F11 MEM medium (1:1 ratio) and incubated for $48 \mathrm{~h}$. Levels of GFP fluorescence were visualized and quantified using a Typhoon scanner (Amersham Bioscience, GE Healthcare Bio-Sciences Corp., Piscataway, NJ, USA). Fluorescence was inversely proportional to the IFN activity. The fluorescence reading of treated cultures was compared with control cultures (no IFN) and presented as relative fluorescence.

\section{Immunofluorescent staining}

GECs were exposed to HIV-1, gp120 or poly I:C (positive control for staining, $25 \mu \mathrm{g} / \mathrm{ml}$ ) and were fixed with $4 \%$ paraformaldehyde (Electron Microscopy Sciences, Hatfield, PA, USA). Cells were stained with primary mouse monoclonal anti-human IRF3 (SL-12, Santa Cruz Biotechnology, Inc.) and Alexa Flour 488-conjugated secondary antibody, followed by propidium iodide nuclear counterstaining. Filters were excised from the polystyrene filter supports, and mounted on glass slides in Vecta Shield Hard Set mounting medium (Vector 
Laboratories, Inc., Burlingame, CA, USA). All samples were imaged on an inverted confocal laser-scanning LSM 510 microscope (Carl Zeiss Canada Ltd, Toronto, ON, USA). Confocal microscope settings for image acquisition and processing were identical between control and treated monolayers and three separate, random images were acquired and analyzed for each experimental condition. Images were analyzed using Image J software for measuring levels of nuclear colocalization of IRF3.

\section{Plasmids and HEK293 transfection assays}

Expression vectors for human TLR2, TLR4, CD14, MD2, $\beta$-galactosidase ( $\beta$-gal) and plasmids were kindly provided by Dr Cynthia Leifer (Cornell University Ithaca, NY, USA). Interferon- $\beta$-luciferase (IFN $\beta$-Luc) and interferon-stimulated response element (ISRE-Luc)-inducible reporter plasmids were a kind gift from Dr John Hiscott and are designed to monitor the activation of IFN $\beta$ pathways. ${ }^{26}$ All plasmids were amplified and purified using Endo-free Midi Prep columns (Qiagen, Toronto, ON, Canada). Transfections for luciferase assay were carried out in HEK293 cell line. Subconfluent HEK293 cells were transfected with $100 \mathrm{ng}$ of pSV $\beta$-gal (internal transfection control for normalizing transfection efficiencies), $100 \mathrm{ng}$ of IFN $\beta$-Luc or ISRE-Luc reporter plasmid (firefly luciferase, experimental reporter), $30 \mathrm{ng}$ of pUNO-hTLR2 or pUNOhTLR4 with or without $30 \mathrm{ng}$ CD14 and pUNO hMD2 expression plasmids and pBABE (empty vector) for a total of $1 \mu \mathrm{g}$ DNA/well. Transfections were completed using Gene Juice transfection reagent (EMD Millipore). At $24 \mathrm{~h}$ post transfection, cells were treated with gp120, Pam3CSK4 (a TLR2 ligand) and LPS (a TLR4 ligand). The cells were subsequently harvested, lysed and luciferase activity was measured using a Stratagene luciferase assay kit (Agilent Technologies) as per the manufacturer's instructions. Activity of pSV- $\beta$-gal luciferase activity was also measured by reporter assay (Luminescent $\beta$-galactosidase detection kit II, Clontech Lab Inc., Mountain View, CA, USA) according to the manufacturer's instruction. The fold increases in IFN $\beta$-luciferase expression or ISRE-luciferase expression (experimental reporters) were normalized with $\beta$-gal expression as an internal control for transfection.

\section{G5 HIV-1 LTR assay}

To measure the effect of various treatments on activation of HIV-1 long terminal repeat (LTR), $10^{6} 1 \mathrm{G} 5$ cells were treated with phorbol 12-myristate 13-acetate (PMA) at $50 \mathrm{ng} / \mathrm{ml}$ (eBioscience) for $24 \mathrm{~h}$ at $37^{\circ} \mathrm{C} .^{27}$ The cells were lysed, and luciferase activity was determined using a luciferase assay (Agilent). To measure the effect of epithelial supernatants containing IFN $\beta$ on 1G5 LTR activation, supernatants were collected from mock or HIV-1-treated cultures. Supernatants were then incubated with anti-IFN $\beta$ antibody $(25 \mu \mathrm{g} / \mathrm{ml}$; Millipore) or anti-TNF $\alpha$ antibody $(25 \mu \mathrm{g} / \mathrm{ml}$; Rockland Immunochemicals) or rabbit isotype IgG control $(25 \mu \mathrm{g} / \mathrm{ml}$; Abcam) for $1 \mathrm{~h}$ before addition to PMA-treated 1G5 cells and luciferase activity was measured after $24 \mathrm{~h}$.

\section{Microarray gene expression and data analysis}

For microarray analysis, $1 \times 10^{5}$ primary genital epithelial cells were exposed to HIV-1 for $48 \mathrm{~h}$ at an MOI of 1 and were processed for RNA isolation using RNAeasy kit (Qiagen). Purified RNA was resuspended in RNase-free water and quantified using a Nanovue spectrophotometer (General Electric, Mississauga, ON, Canada). RNA bioanalysis, microarray chip hybridization and processing were performed by the Center for Applied Genomics Facility at Hospital for Sick Kids (Toronto, Ontario, Canada). DNA microarray analysis was carried out using the Affymetrix Human Genome ST 2.0 array (Affymetrix, Santa Clara, CA, USA) according to the manufacturer's instructions (Agilent Technologies). The .CEL data files generated by the Affymetrix microarray were analyzed using GeneSpring GX version 13.0 (Agilent Technologies). Fold changes $(>2.0)$ in gene expression and gene ontology annotations were determined.

\section{Quantitative real-time PCR}

Quantitative real time-PCR (qRT-PCR) was performed using gene-specific primers listed in Table 1 and SYBR Green PCR

Table 1 Real-time PCR primers for different interferon-stimulated genes (ISGs)

\begin{tabular}{lccc}
\hline Name & Accession no. & 5' Sequence & 3' Sequence $^{\prime}$ \\
\hline MX1 & BC032602 & CAGCACCTGATGGCCTATCA & ACGTCTGGAGCATGAAGAACTG \\
ISG15 & M 13755 & ACTCATCTTTGCCAGTACAGGAG & CAGCATCTTCACCGTCAGGTC \\
IFIT1 & BC007091 & GCAGCCAAGTTTTACCGAAG & GCCTATCTGGTGATGCAGT \\
IFIT3 & AK297137 & AGTCTAGTCACTTGGGGAAC & ATAAATCTGAGCATCTGAGAGTC \\
IFI44L & BC015932 & ATGACCCGGCTTTGAGAAGT \\
RSAD2 & AF442151 & GTATAGCATATGTGGCCTTGCTTACT & GATCAGGCTTCCATTGCTC \\
OAS1 & AY730627 & AGGTTCTGCAAAGTAGAGTTGC & TAGAAGGCCAGGAGTCAGGA \\
OAS2 & BC049215 & CAGGCAGAAGAGGACTGGAC & GGAAGAAATTTGCGGATGA \\
OAS3 & AB044545 & CTTCTGCCTTTGGCTTTTG & CTCCTTCCACAACCCCTGTA \\
BST2 & AK303593 & GGAAACCCAAGCCACAAGT & GTGGCATTTGCCTTGTTTTT \\
GAPDH & NM_002046 & ACAGTCAGCCGCATCTTCTTTTGC & TTGAGGTCAATGAAGGGGTC \\
\hline
\end{tabular}


mix according to the manufacturer's manual (FroggaBio, Toronto, ON, Canada). qRT-PCR was performed using StepOne Plus ${ }^{\mathrm{TM}}$ Real-Time PCR System (Thermo Fisher, Burlington, ON, Canada). Samples were run in triplicate and all data were normalized to glyceraldehyde 3-phosphate dehydrogenase (GAPDH) mRNA expression as an internal control.

\section{Statistical analysis}

GraphPad Prism Version 4 (GraphPad Software, San Diego, CA, USA) was used to compare three or more means by oneway analysis of variance (ANOVA) for analyzing different treatments at the same time and two-way ANOVA for comparing treatments with their specific controls. To compare two different treatment means, unpaired $t$-test was used. When an overall statistically significant difference was seen $(P<0.05)$, Bonferroni post-test was performed to adjust the $P$-value for multiple comparisons. $P$-values for each analysis are indicated in figure legends.

\section{RESULTS}

Antiviral responses induced by endometrial epithelial cells in response to HIV gp120

To examine the innate antiviral response of epithelial cells from upper genital tract, confluent monolayers of endometrial epithelial cells were exposed to HIV-1 $\left(10^{5} /\right.$ well) or recombinant gp120 protein $(100 \mathrm{ng} / \mathrm{ml})$, or an $e n v$-deleted mutant that lacks the HIV-1 viral envelope precursor, gp160, ${ }^{18,28}$ and supernatants were analyzed for IFN $\beta$ by enzyme-linked immunosorbent assay (ELISA). The results showed that supernatants collected from monolayers treated with HIV-1 or gp120 contained significantly higher amount of IFN $\beta$ as compared with supernatants from mock treatment (Figure 1a). IFN $\beta$ level was much higher in basolateral supernatants compared with apical supernatants, demonstrating preferential secretion of IFN $\beta$ from the basal interface of the epithelium (Figure 1a). The env-deleted HIV-1 mutant failed to induce IFN $\beta$ in endometrial GECs, indicating that HIV envelope is required to induce antiviral responses in endometrial GECs (Figure 1a). The levels of IFN $\beta$ produced by different donor tissues was variable based on the tissue: baseline (range $20-80 \mathrm{pg} / \mathrm{ml}$, $n=6$ ), apical supernatants following HIV-1/gp120 treatment $(140-600 \mathrm{pg} / \mathrm{ml}, n=6)$ and basolateral supernatants (200$2185 \mathrm{pg} / \mathrm{ml}, n=6)$ but the increased responses to gp120 and HIV-1 was similar in all tissues. To confirm the role of gp120 in IFN $\beta$ production by endometrial GECs, viral preparations as well as recombinant gp120 were incubated with anti-gp120 neutralizing antibody (2G12 clone) or isotype antibody before exposure to endometrial GECs. Supernatants collected $24 \mathrm{~h}$ post treatment showed that gp120 neutralization reduced induction of IFN $\beta$ to baseline levels (Figures 1b-d). All together, these results indicate that HIV-1 gp120 is directly responsible for induction of an IFN $\beta$ response in endometrial GECs.

Next, we examined the time kinetics of induction of IFN $\beta$ in response to HIV-1 and gp120. Poly I:C was used as a positive control. Endometrial GECs were exposed to different treatments and supernatants were collected hourly for 1-6h and at $24 \mathrm{~h}$. IFN $\beta$ was significantly induced in apical supernatants at $6 \mathrm{~h}$ time point in response to HIV-1 and gp120, but significantly higher induction was observed in basolateral supernatants at earlier time points (Figures le and $\mathrm{f}$ ).

In order to examine biological activity of gp120-mediated IFN, we used a VSV-GFP assay that assesses the presence of biologically active IFN. ${ }^{5,29}$ Using this assay, we measured the ability of VSV-GFP to replicate in Vero cells, in the presence of supernatants from UV-inactivated HIV-1 or gp120-treated endometrial GEC cultures. The presence of biologically active type I IFN was confirmed in supernatants from UV-inactivated HIV-1 and gp120-treated GEC cultures by the significant decrease in replication of VSV-GFP (Figures $1 \mathrm{~g}$ and $\mathrm{h}$ ). Neutralization of recombinant gp120 with neutralizing antibodies completely abrogated type I IFN-mediated protection against VSV-GFP replication, whereas addition of isotype antibody did not influence the induction of biologically active type I IFN production (Figures $1 \mathrm{~g}$ and $\mathrm{h}$ ).

\section{Antiviral responses induced in primary endocervical cells by HIV-1}

To examine whether HIV-1 also induced antiviral responses in primary endocervical epithelial cells, similar to endometrial epithelial cells, we exposed primary endocervical epithelial cultures to medium (mock) or HIV-1 ( $10^{5}$ IU/well) for $24 \mathrm{~h}$ and measured TER as a measure of epithelial barrier function tumor necrosis factor- $\alpha$ (TNF $\alpha)$ and IFN $\beta$ production. Similar to the previously reported effect of HIV-1 in endometrial epithelial cells, HIV-1 exposure impaired the barrier function in endocervical epithelial cells and induced significant production of TNF $\alpha$ and IFN $\beta$, although induction of IFN $\beta$ appeared to be less potent compared with endometrial GECs (Supplementary Figures 1a-d). As the effects of HIV-1 on barrier disruption, TNF $\alpha$ and IFN $\beta$ production were analogous in primary endometrial and endocervical epithelial cells, further experiments were conducted on primary endometrial epithelial cells that were more abundantly available and because the larger amount of tissue provided bigger cultures.

\section{Role of IFN $\beta$ in HIV-mediated barrier impairment}

Previously, we reported that exposure to HIV in endometrial and cervical epithelial cells resulted in impairment of epithelial barrier primarily because of the upregulation of inflammatory cytokines, particularly TNF $\alpha^{46}$. However, the effect of IFN $\beta$ on gp120-mediated barrier disruption has not been examined. Therefore, we next examined the role of IFN $\beta$ in HIV-1 gp120mediated barrier disruption. Confluent monolayers of endometrial GECs were treated with medium (mock) or HIV-1 in the presence or absence of human anti-IFN $\beta$ antibody, human anti-TNF $\alpha$ antibody or isotype antibody. At $24 \mathrm{~h}$ post treatment TER was measured and compared with baseline values. As expected, HIV-1 significantly decreased the TER, indicating barrier disruption, but the presence of TNF $\alpha$ neutralizing antibodies ameliorated the HIV-mediated barrier disruption, whereas addition of anti-IFN $\beta$ neutralizing 

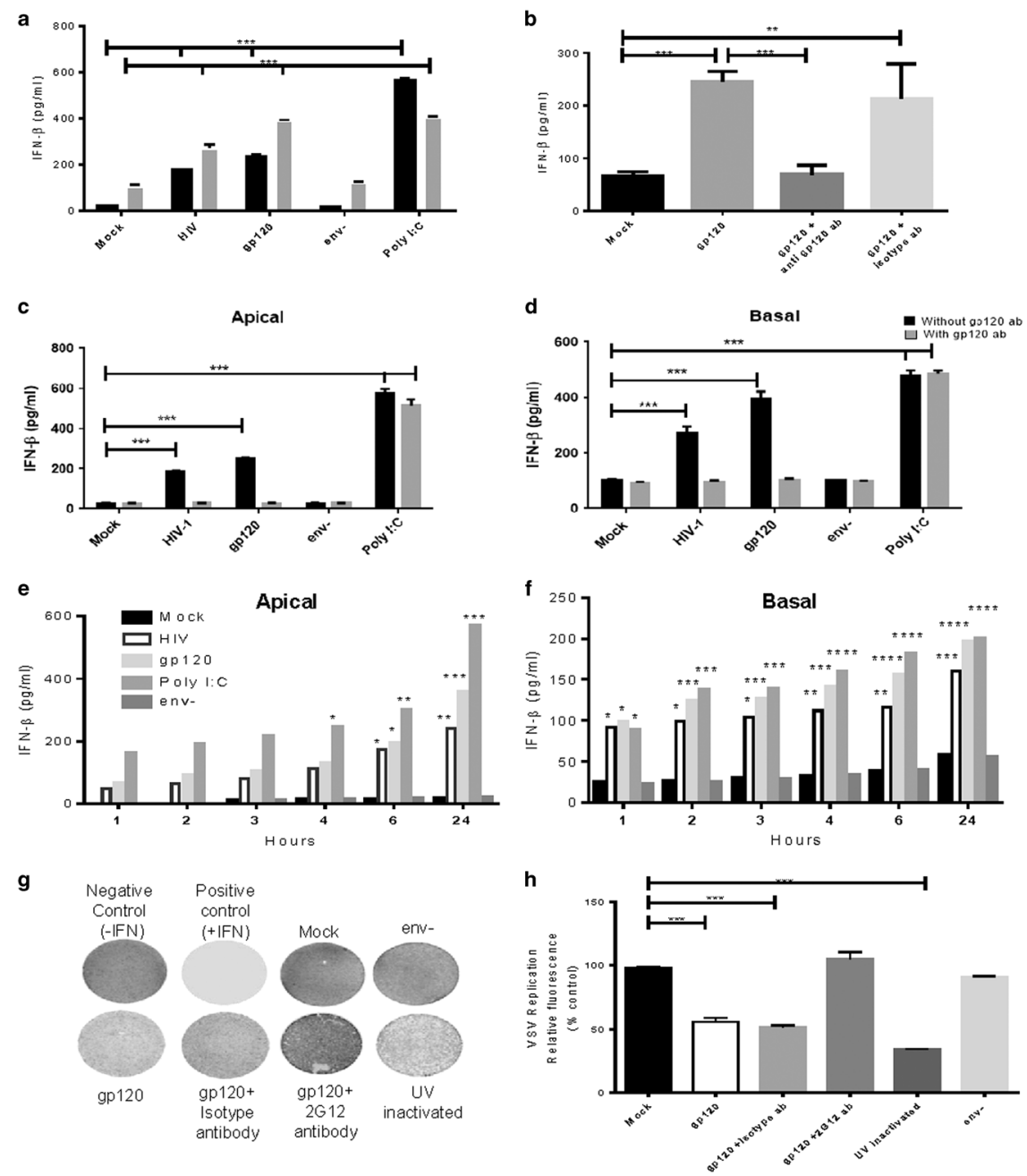

Figure 1 Primary endometrial epithelial cells respond to HIV-1 envelope gp120 by production of IFN $\beta$. (a) Confluent monolayers of primary

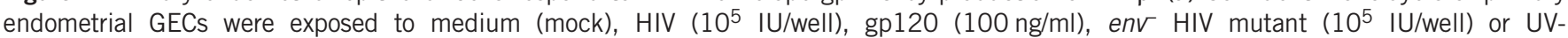
inactivated HIV for $24 \mathrm{~h}$ and supernatants were analyzed for IFN $\beta$ production. IFN $\beta$ was measured by ELISA in both apical (black bars) and basolateral (gray) supernatants. Endometrial GECs produce significant amounts of IFN $\beta$ in response to HIV, gp120 and poly I:C when compared with mock-treated cells. $N=4$. (b) Endometrial GECs were exposed to gp120 alone or with anti-gp120 neutralizing (2G12) or isotype antibody for $24 \mathrm{~h}$, apical supernatants were collected and analyzed for IFN $\beta$ production by ELISA. $N=2$. (c, d) Endometrial GECs were exposed for $24 \mathrm{~h}$ to medium (mock), HIV, gp120, env HIV mutant or poly I:C, with or without anti gp120 neutralizing antibody. IFN $\beta$ production in supernatants was determined without gp120 antibody (black bars) or following treatment with anti gp120 antibody (gray bars). (e, f) Time course for IFN $\beta$ production was analyzed in supernatants collected from endometrial GECS after exposure to medium (mock), HIV, gp120, poly I:C, env HIV mutant for 1, 2, 3, 4, 6 and $24 \mathrm{~h}$. IFN $\beta$ production in apical (c) and basal (d) supernatants was measured by ELISA. (g) Endometrial GECs were exposed to mock or env- mutant or gp120 alone or in the presence of either isotype antibody or gp120 neutralizing antibody or UV-inactivated HIV-1 for $24 \mathrm{~h}$. Supernatants were tested by VSV-GFP assay on Vero cells to determine biological activity of type I IFNs. VSV-GFP was added to Vero cells without a pretreatment (negative control), recombinant IFN $\beta(100 \mathrm{pg} / \mathrm{ml})$ added to Vero cells before VSV infection (positive control) or supernatants collected from treatments done in above experiments. GFP fluorescence, an indicator of VSV-GFP infection, was quantified by scanning the plates on a Typhoon Biomolecular imager. Images are representative of three separate experiments conducted in triplicate. (h) Quantitative representation of VSV-GFP fluorescence. Each experimental condition was compared with negative control and calculated as percent of negative control. Data shown represent mean \pm s.e.m. of three separate experiments from cells isolated from three individual tissues. ${ }^{*} P<0.05$, ${ }^{* *} P<0.01$ and $* * * P<0.001$. ELISA, enzyme-linked immunosorbent assay; GEC, genital epithelial cell; GFP, green fluorescent protein; IFN $\beta$, interferon- $\beta$; poly I:C, polyinosinic:polycytidylic acid; UV, ultraviolet; VSV, vesicular stomatitis virus.

antibody significantly exacerbated the HIV-mediated barrier disruption (Figure 2a). Supernatants collected from these cultures confirmed the protective role of IFN $\beta$, as neutralization of IFN $\beta$ resulted in upregulation of TNF $\alpha$ (Figure $2 b$ ) that correlated with increase in barrier impairment compared with effect of HIV-1 alone. Neutralization of TNF $\alpha$ resulted in upregulation of IFN $\beta$ production that correlated with enhanced protection of barrier (Figure 2c). These studies indicate that IFN $\beta$ produced by GECs opposes the effect of TNF $\alpha$ and plays a protective role on HIV-1-mediated barrier disruption. 


\section{Induction of IFN $\beta$ by HIV-1 gp120 is mediated through IRF3 activation}

Induction of IFN $\beta$ is typically mediated through activation of transcription factor, IRF3. Therefore, we next examined the involvement of IRF3 in IFN $\beta$ production by GECs in response to HIV-1. GEC monolayers were exposed to HIV-1 and gp120 for different lengths of time and fixed and stained for IRF3. Nuclear translocation of IRF3 was observed at $1 \mathrm{~h}$ post exposure to HIV-1 or gp120. Exposure to gp120 in the
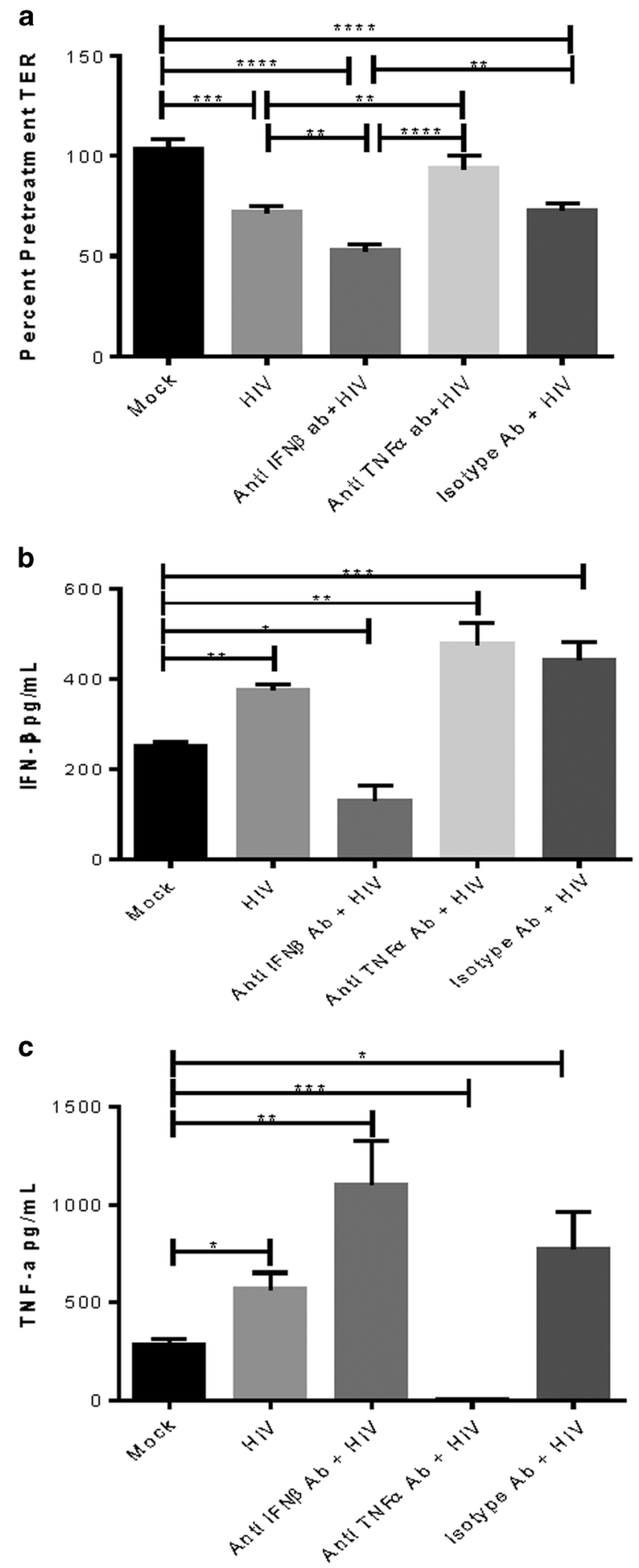

presence of anti-gp120 antibodies (2G12) inhibited IRF3 translocation (Figures $3 \mathrm{a}$ and $\mathrm{b}$ ). Peak translocation of IRF3 was observed at $2 \mathrm{~h}$ post exposure with HIV-1 or gp120 (Figure 3c). The TLR3 ligand poly I:C, which is known to activate IRF3, was used as a positive control. These results indicate that activation and translocation of IRF3 is a direct consequence of exposure to $\mathrm{HIV}_{-1}$ viral envelope protein, gp120.

HIV-1 or gp120-mediated IFN $\beta$ production is blocked by inhibition of IRF3

As IFN $\beta$ can be induced through transcription factors other than IRF3, we next examined the contribution of IRF3 pathway towards gp120-mediated induction of IFN $\beta$ by inhibiting IRF3. BX795, a specific inhibitor of TBK-1/ IKKe phosphorylation, an upstream event critical in activation of IRF3, was used for inhibiting the IRF3 pathway. ${ }^{30}$ GEC monolayers were pretreated with medium or BX795 for $1 \mathrm{~h}$ before exposure with gp120 for $24 \mathrm{~h}$. Supernatants were collected and analyzed for IFN $\beta$ production by ELISA. BX795 almost completely inhibited gp120-mediated induction of IFN $\beta$ production (Figures 3d and e), indicating that activation of IRF3 was necessary for the induction of IFN $\beta$ by gp120. Interestingly, whereas IFN $\beta$ was almost completely inhibited by BX795, the levels of proinflammatory cytokines, including TNF- $\alpha$ and IL- $1 \alpha$, were increased in the presence of BX795, a likely effect of cross-regulation between IFN $\beta$ and pro-inflammatory cytokine pathways $^{31}$ (Supplementary Figure 2).

To confirm that BX795 was inhibiting IRF3 activation, endometrial GEC monolayers were stained for IRF3 after treatment with and without BX795 before HIV-1 and gp120 treatment. Confocal microscopy results revealed that BX795 inhibited HIV-mediated and gp120-mediated IRF3 activation and nuclear translocation (Figures $3 \mathrm{f}$ and $\mathrm{g}$ ). Treatment with BX795 in mock and HIV-1-treated cultures did not have any effect on the GEC viability, as seen by TER (Figure $3 \mathrm{~h}$ ), a measurement of epithelial tight junctions used as indicators of the barrier function and overall integrity of monolayer. As expected, HIV-1 treatment decreased TERs significantly compared with mock treatment and the presence of BX795 exacerbated this effect (Figure $3 \mathrm{~h}$ ).

Figure 2 Role of IFN $\beta$ in HIV-mediated barrier impairment. Confluent monolayers of primary endometrial GECs were exposed to medium (mock) and HIV-1 (10 IU/well) in the absence or presence of human anti-IFN $\beta$ antibody, anti-TNF $\alpha$ antibody or isotype control antibody. (a) Epithelial barrier function was determined by measurement of TER at $24 \mathrm{~h}$ post treatment and shown as ratio of pretreatment values. Supernatants were collected after $24 \mathrm{~h}$ of treatments and analyzed for (b) TNF $\alpha$ and (c) IFN $\beta$ by ELISA. Data shown are representative of two separate experiments done on tissues taken from two different donors and show mean \pm s.e.m. of treatments run in duplicate. ${ }^{*} P<0.05$, ${ }^{* *} P<0.01,{ }^{* * *} P<0.001$ and $* * * * P<0.0001$. ELISA, enzymelinked immunosorbent assay; GEC, genital epithelial cell; IFN $\beta$, interferon- $\beta$; TER, transepithelial resistance; TNF $\alpha$, tumor necrosis factor- $\alpha$. 
Previous studies have shown that in addition to IRF transcription factors (IRF3/7), NF- $\mathrm{KB}$ may also play a critical role in IFN $\beta$ expression. ${ }^{32}$. Previously, we have shown that gp120 treatment leads to NF- $\mathrm{BB}$ translocation in GECs and blocking it with pyrrolidine dithiocarbamate inhibits production of inflammatory cytokines including TNF $\alpha .{ }^{19}$ When GEC a
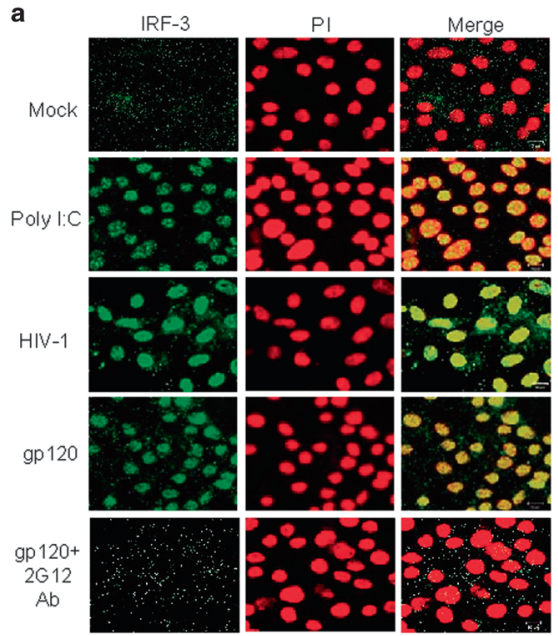

d

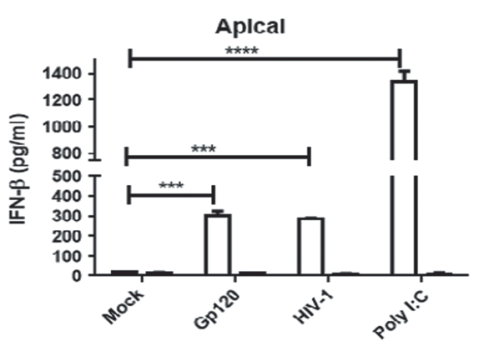

$\mathbf{f}$

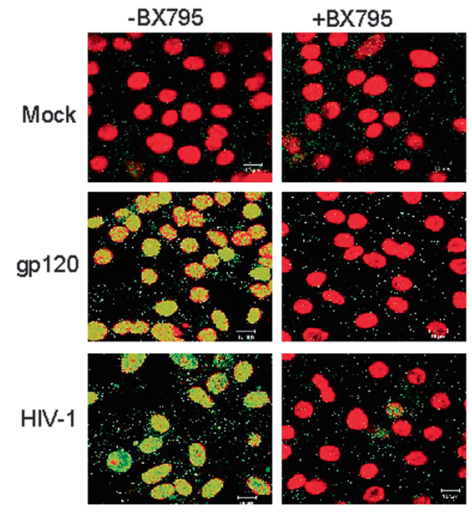

b

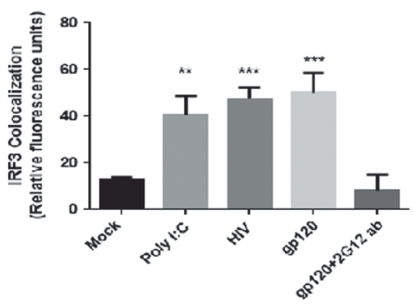

c
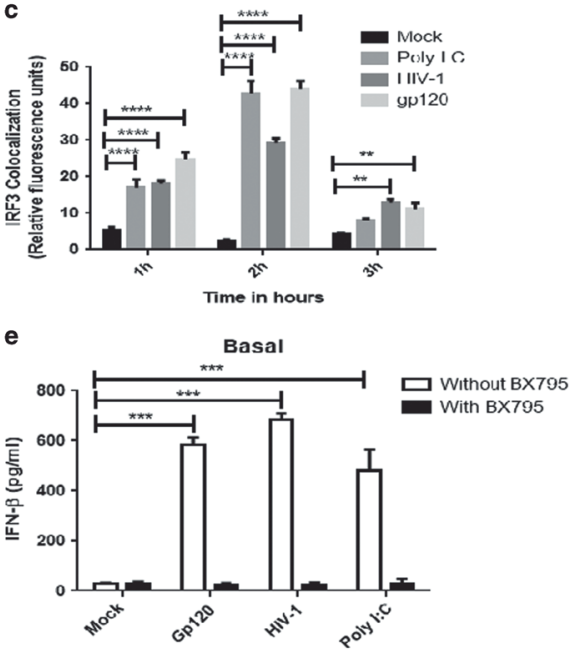

g

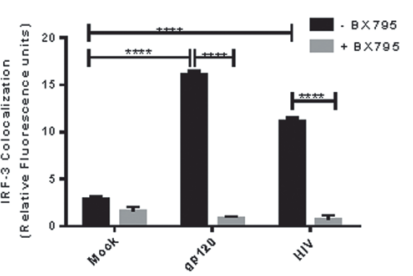

h

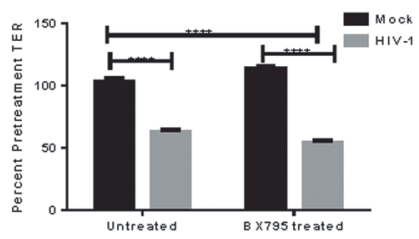

Figure 3 Induction of IFN $\beta$ in endometrial GECs by HIV-1 gp120 is mediated through IRF3. Endometrial GECs were exposed to medium or poly I:C, HIV-1 (10 IU/well) or gp120 (100 ng/ml alone or with anti-gp120 neutralizing antibody) for $1-3 \mathrm{~h}$. Cells were fixed and stained for the IRF3 (green fluorescence). Nuclear counterstaining (red fluorescence) was achieved using PI. Images were captured by a laser-scanning confocal microscopy. (a) Representative images are shown at $2 \mathrm{~h}$ time point from one of three separate experiments. Magnification $\times 1260$. (b) IRF3 translocation and nuclear colocalization was measured by Image J software and presented as relative light units. (c) Time kinetics of IRF3 colocalization following treatment of endometrial GECs with medium or poly I:C (positive control), HIV-1 or gp120. (d, e) Endometrial GECs were incubated with the IRF3 inhibitor, BX795 (1 $\mu \mathrm{M})$ for $1 \mathrm{~h}$, before exposure with gp120, HIV-1 or poly I:C (positive control). Supernatants were collected after $24 \mathrm{~h}$ and assayed by ELISA. Results showed IFN $\beta$ production in apical (d) and basolateral supernatants (e). (f) Endometrial GECs were treated with BX795 for $1 \mathrm{~h}$ before gp120 or HIV-1 exposure for $2 \mathrm{~h}$. The cells were fixed and stained for IRF3 and nuclei. Images were captured by laser-scanning confocal microscopy. Magnification: $\times 1260$. (g) Colocalization was measured by image $\mathrm{J}$ software and represented in a bar diagram. $\mathbf{h}$ Endometrial GECs were preincubated with BX795 or media (mock) for $1 \mathrm{~h}$ and TERs were measured pretreatment and after $24 \mathrm{~h}$ of treatment with mock or HIV-1 to check whether BX795 was affecting HIV-1-mediated barrier disruption. Images are representatives of three separate experiments from cells isolated from three individual tissues. ${ }^{*} P<0.05,{ }^{* *} P<0.01,{ }^{* *} P<0.001$ and ${ }^{* * * *} P<0.0001$. ELISA, enzyme-linked immunosorbent assay; GEC, genital epithelial cell; IFN $\beta$, interferon- $\beta$; IRF3, interferon regulatory factor 3; PI, propidium iodide; poly I:C, polyinosinic:polycytidylic acid; TER, transepithelial resistance. 
monolayers were treated with pyrrolidine dithiocarbamate, before treatment with gp120, there was a small but significant decrease in IFN $\beta$ produced by GECs in response to gp120 that indicated that NF- $\mathrm{BB}$ likely enhanced the gp120-mediated IFN $\beta$ production (Supplementary Figure 3a). However, blocking NF- $\kappa \mathrm{B}$ with pyrrolidine dithiocarbamate did not have any effect on IRF3 translocation (Supplementary Figure 3b). These results indicate that IRF3 activation is the primary pathway for IFN $\beta$ induction in the GECs in response to gp120, but NF- $\kappa B$ may also play a minor role in enhancing IFN $\beta$ production.

\section{HIV-1 gp120-mediated IRF3 activation and IFN $\beta$ induction occurs via TLR2 signaling pathway}

Previously, we showed that HIV-1 can induce proinflammatory cytokines by signaling through TLR2 and TLR4. ${ }^{19}$ Therefore, we next examined whether TLR2 and 4 played any role in IFN $\beta$ induction. TLR5 was included as a control in these experiments, as these three TLRs are primarily responsible for recognizing pathogen-associated glycoproteins at the surface of cells and inducing intracellular signaling. ${ }^{33}$ TLRs 1 and 6 are also associated with cell-surface protein recognition, but act in association with TLR2. We first determined whether TLRs 2, 4 or 5 were associated with gp120-mediated induction of IFN $\beta$. GECs were pretreated with neutralizing antibodies against TLR2, TLR4 and TLR5 before gp120 exposure. Supernatants were collected and analyzed by IFN $\beta$ ELISA. IFN $\beta$ production was completely blocked in the presence of only TLR2 antibody (Figure $4 \mathrm{a}$ ). In contrast, pretreatment of GECs with isotype control, TLR4 or TLR5 neutralizing antibody did not have any effect on gp120-mediated IFN $\beta$ production (Figure $4 \mathrm{a}$ ). Pam3CSK4 (synthetic triacylated lipopeptide), a TLR2 ligand, and FimH (Fimbriae protein), a TLR4 ligand, that are known to induce these pathways in GECs were used as controls to verify the specificity of the antibodies. ${ }^{34,35}$

\section{HIV-1 gp120-mediated IRF3 translocation is blocked in the presence of TLR2 antibody}

As the induction of IFN $\beta$ production in GECs was seen to be mediated through TLR2 pathways, we next determine whether neutralizing antibodies to TLR2 abrogated gp120-mediated activation and nuclear translocation of IRF3. TLR4 and TLR5 antibodies were used as controls to confirm the specificity of the interaction. GEC monolayers were incubated with antibodies against TLR2, TLR4, TLR5 or isotype control for $1 \mathrm{~h}$ before treatment with gp120. After $1 \mathrm{~h}$ of gp120 exposure, cells were fixed and stained for IRF3. IRF3 nuclear translocation was found to be blocked following gp120 treatment in the presence of TLR2 antibody, but not TLR4, TLR5 or isotype antibodies (Figures 4b and c). These results confirm that gp120-mediated IFN $\beta$ production and IRF3 activation was mediated via TLR2 signaling pathway.

\section{Gp120 can induce IFN $\beta$ and ISRE genes through TLR2}

Our previous results had shown that gp120 activates proinflammatory cytokines and NF- $\mathrm{BB}$ pathway via both TLR2 and TLR4, yet experiments in primary endometrial GEC monolayers indicated that HIV-1 gp120 induced IFN $\beta$ via TLR2 pathway alone. Therefore, to confirm the primary epithelial cell results, we used a cell line-based expression system in the kidney embryonic cell line, HEK293, that has been used extensively for testing TLR functioning. ${ }^{36,37}$ HEK293 cells were either transfected with an IFN $\beta$-luciferase or ISREluciferase reporter plasmid and a TLR2 or TLR4 expression plasmid and subsequently stimulated with gp120 or with known TLR ligands. TLR4 plasmid was also cotransfected with MD2 and CD14 plasmids as these proteins are required for LPS-mediated TLR4 signaling. ${ }^{38}$ Gp120 activated IFN $\beta$ and ISRE expression significantly only in TLR2-transfected cells compared with mock treatment as measured by luciferase activity (Figures 5a, b, e and f), whereas there was no induction of luciferase activity by gp120 in cells transfected by TLR4 expression plasmid with and without MD2 and CD14 (Figures $5 \mathrm{c}, \mathrm{d}, \mathrm{g}$ and $\mathrm{h}$ ). The absence of gp120 in env $\mathrm{v}^{-}$mutant treatment failed to activate IFN $\beta$ and ISRE expression in both TLR2 and TLR4 cotransfected cells, confirming the specific role of gp120 in activation of IFN $\beta$ through TLR2 pathway.

\section{Cell surface heparan sulfate is required for gp120-mediated IFN $\beta$ production}

Previously, we have shown that HIV-1 gp120-mediated induction of inflammatory response was activated through TLR2 and TLR4 pathway and required presence of heparan sulfate on cell surface. ${ }^{19}$ Therefore, we next examined whether heparan sulfate was also required for the induction of IFN $\beta$ by gp120. Confluent GEC monolayers were first treated with heparinase III to remove heparan sulfate from the cell surface or exogenous heparan sulfate was added before treating cells with HIV-1 gp120 or poly I:C, a TLR3 ligand that does not require presence of heparan sulfate to activate the TLR3 pathway and leads to induction of IFN $\beta$. GEC cultures that were treated with media instead of gp120 acted as negative controls. The results indicated that gp120 required the presence of heparan sulfate on the cell surface in order to induce IFN $\beta$ production as removal of heparan sulfate by heparinase III decreased the IFN $\beta$ production in GECs to baseline (Figures $6 a$ and $b$ ). Cells treated with heparinase III followed by addition of exogenous heparan sulfate restored induction of IFN $\beta$ production in response to gp120, confirming that heparan sulfate is required for gp120 to initiate the IFN $\beta$ signaling pathway.

\section{Gp120-mediated IFN $\beta$ responses by endometrial GECs reduces HIV-1 infection}

Thus far, our results indicated that HIV-1 gp120 was inducing upregulation of IFN $\beta$ from GECs from upper genital tract via TLR2 pathway through activation of IRF3. However, we have previously reported that gp120 also activates the production of proinflammatory cytokines via the NF- $\kappa \mathrm{B}$ pathway through TLR2 and TLR4 activation. ${ }^{19}$ As the proinflammatory cytokines are known to enhance HIV-1 infection and replication whereas the IFN $\beta$ pathway has antiviral effects, we next examined the functional significance of IFN $\beta$ production in response to HIV-1 gp120 with respect to HIV-1 infection and replication. 


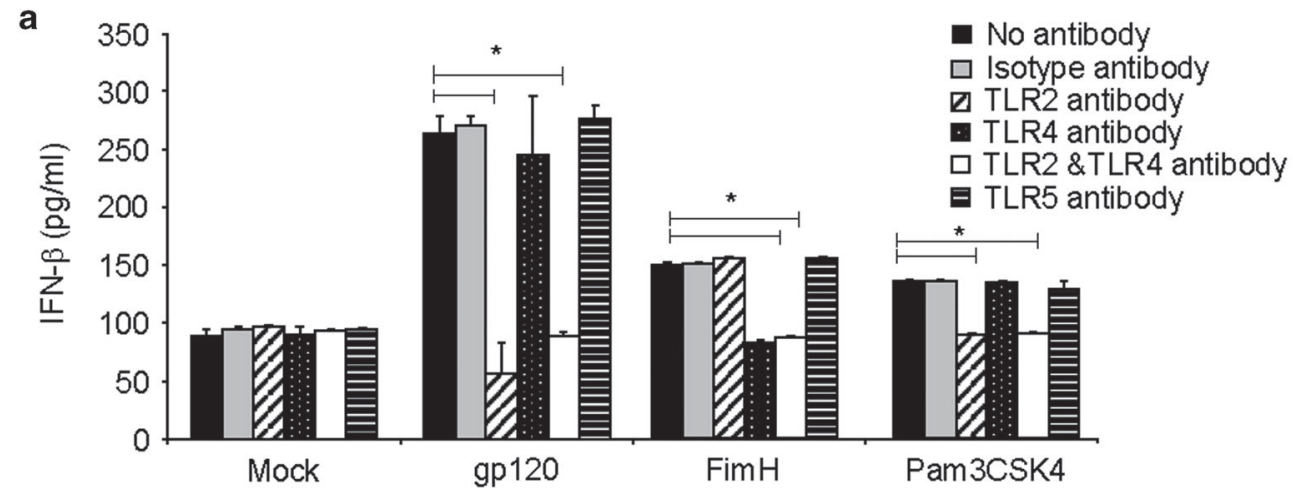

b

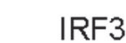

Untreated

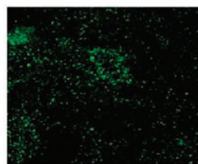

gp120
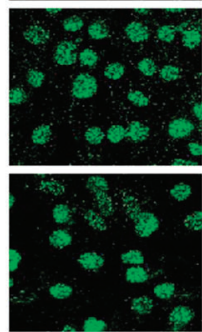

gp120+

Isotype

antibody
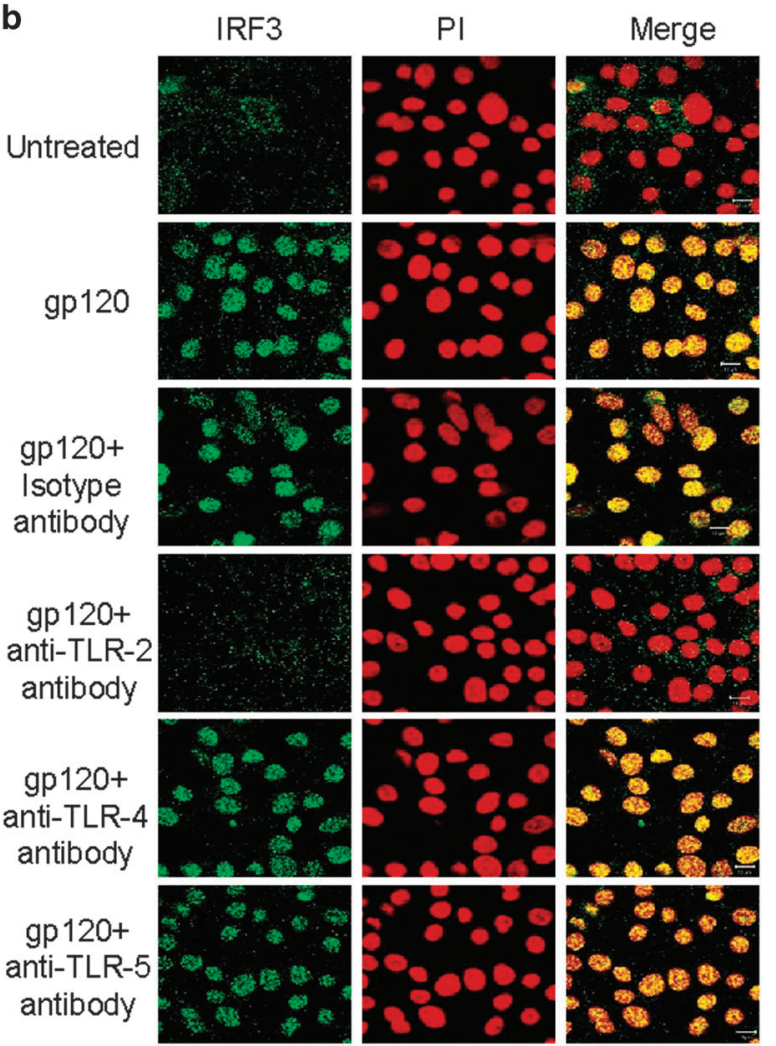

C

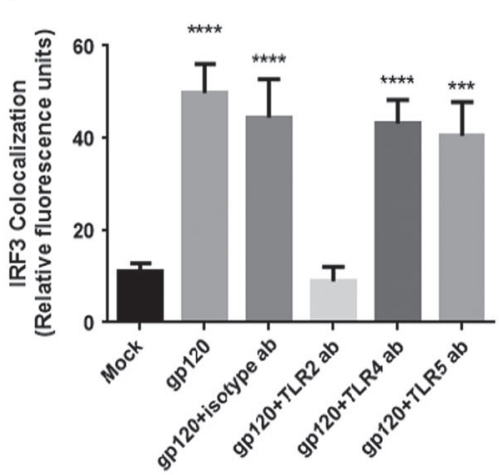

Figure 4 Neutralization of TLR2 blocks gp120-mediated IFN $\beta$ production and IRF3 activation. (a) Endometrial GECs were pretreated with neutralizing antibodies against TLR2, TLR4, TLR5 or isotype control antibodies (all at $10 \mu \mathrm{g} / \mathrm{ml}$ ) before exposure to gp120 (100 ng/ml) or mock treatment (media). FimH and Pam3CSK4 were used as positive controls for activation of TLR4 and TLR2, respectively. Supernatants were collected after $24 \mathrm{~h}$ and analyzed by ELISA for IFN $\beta$ production. Data shown are mean+s.d. and representative of three separate experiments done on cells isolated from three different tissues. (b) Epithelial monolayers were fixed after $2 \mathrm{~h}$ of exposure of gp120 with and without pretreatment with neutralizing antibodies against TLR2, TLR4, TLR5 or isotype control antibody and stained for IRF3. Propidium iodide was used to stain nuclei. Images were captures by a laser-scanning confocal microscopy. Magnification $\times 1260$. Images are representative of one of three separate experiments done on cells isolated from three different tissues. (c) Quantitation of IRF3 colocalization were done by Image J software and presented in the graph. Significance was calculated by one-way ANOVA and IRF3 colocalization in all treatments were compared with mock treatment. ${ }^{*} P<0.05,{ }^{* * *} P<0.001$ and ${ }^{* * *} P<0.0001$. ANOVA, analysis of variance; ELISA, enzyme-linked immunosorbent assay; GEC, genital epithelial cell; IFN $\beta$, interferon- $\beta$; IRF3, interferon regulatory factor 3; TLR, Toll-like receptor.

We tested the effect of IFN $\beta$ produced by GECs on HIV-1 infection by examining the effect of supernatants collected from primary female GECs on HIV-1 infection of TZM-bl indicator cells. Supernatants collected from mock and gp120exposed GECs were added to pre-seeded TZM-bl cells before HIV-1 infection and rate of infection was measured by luciferase activity. The recombinant human IFN $\beta(100 \mathrm{pg} / \mathrm{ml}$; $n=6$ ) was used as a positive control. The results showed that adding recombinant IFN $\beta$ and supernatants collected from GEC exposed to gp120 significantly reduced luciferase activity, an indicator of decreased HIV-1 infection in TZM-bl cells because of antiviral activity (Figure 7a). To confirm that the 

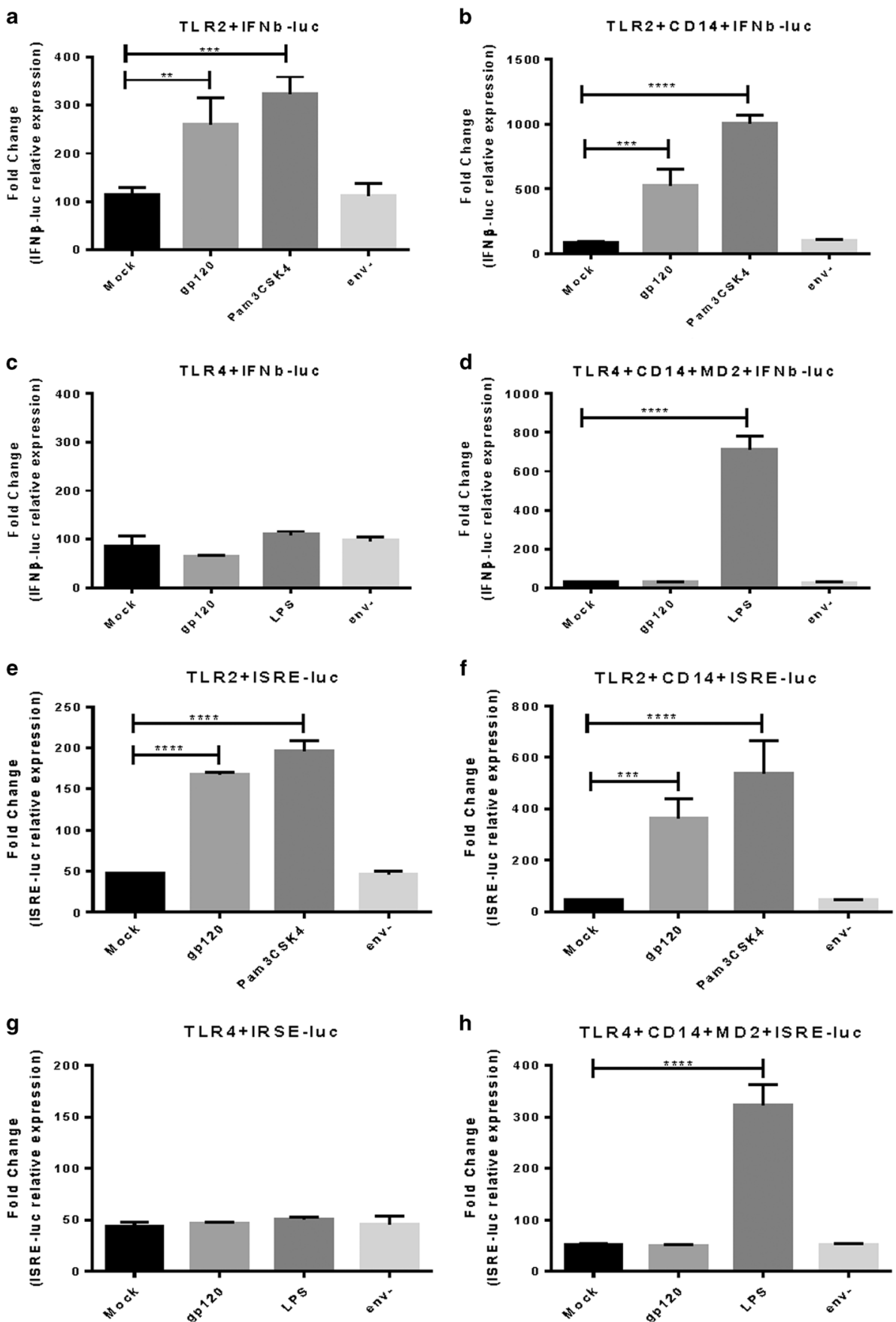

Figure 5 Gp120 induces IFN $\beta$ and ISRE through activation of TLR2. HEK293 cells were transiently transfected with either IFN $\beta$-luciferase (a-d) or ISRE-luciferase $(\mathbf{e}-\mathbf{h})$ reporter in combination with TLR2 (a, b, e, f) or TLR4 (c, d, $\mathbf{g}, \mathbf{h})$ expression plasmid alone or in combination with CD14 and or MD2 expression plasmid. At $24 \mathrm{~h}$ after transfection, cells were stimulated with gp120 (100 ng/ml), or env ${ }^{-}$ mutant $\left(10^{5} \mathrm{IU} /\right.$ well) or $10 \mu \mathrm{g} / \mathrm{ml} \mathrm{Pam3CSK4}$ (positive control for TLR2), or (0.1 mg LPS (positive control for TLR4) or with medium (mock, negative control). Cells were disrupted, and luciferase activity was measured $16 \mathrm{~h}$ after stimulation and normalized to $\beta$-galluciferase activity. Data shown are representative of three individual experiments, each performed in triplicate. Data are represented as mean \pm s.d. ${ }^{* *} P<0.01,{ }^{* *} P<0.001$ and ${ }^{* * * *} P<0.0001$. $\beta$-gal, $\beta$-galactosidase; GEC, genital epithelial cell; IFN $\beta$, interferon- $\beta$; IRF3, interferon regulatory factor 3; ISRE, interferon-stimulated response element; LPS, lipopolysaccharide; TLR, Toll-like receptor.

decreased infection was due to IFN $\beta$ present in epithelial supernatants, TZM-bl cells were treated with supernatants from GECs exposed to gp120 in the presence or absence of IFN $\beta$ antibody or isotype control before infection with HIV-1. Neutralizing IFN $\beta$ with anti-IFN $\beta$ antibody in both recombinant
IFN $\beta$ and gp120-exposed GEC supernatant resulted in loss of antiviral activity, resulting in increased infection similar to that seen in controls where TZM-bl cells were directly infected with HIV-1 (Figure 7a). Mock-infected GECs did not show any significant antiviral activity and treatment with isotype control 

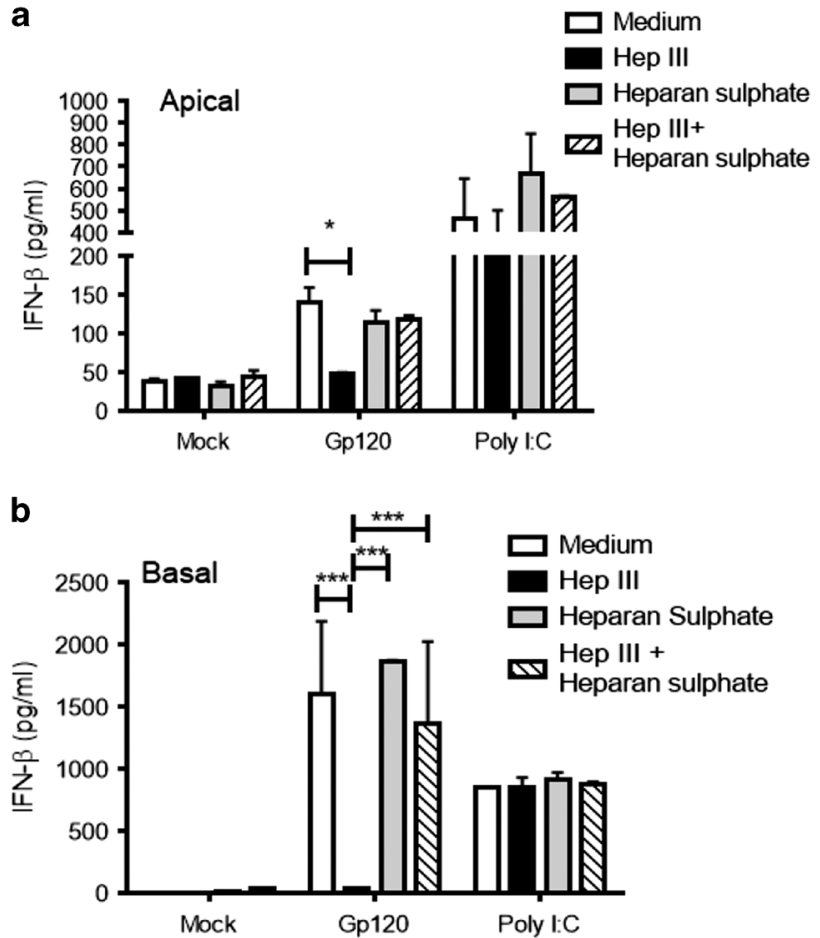

Figure 6 Cell surface heparan sulfate is necessary for gp120mediated induction of IFN $\beta$. Confluent endometrial epithelial monolayers were pretreated with heparinase III for $1 \mathrm{~h}$ before exposure with medium (mock), gp120 (100 ng/ml) or treated with heparan sulfate in the presence of gp120 treatment. Apical (a) and basolateral (b) supernatant were collected and analyzed for IFN $\beta$ production. Data are representative of three experiments from three different tissues, each performed in triplicate. Data are represented as mean \pm s.d. ${ }^{*} P<0.05,{ }^{* * *} P<0.001$. IFN $\beta$, interferon $-\beta$.

antibody instead of anti-IFN $\beta$ antibody did not have any effect on luciferase activity.

These results show that IFN $\beta$ produced by epithelial cells from the upper genital tract in response to HIV-1 exposure can exert an antiviral effect by decreasing HIV-1 infection.

\section{Gp120-mediated IFN $\beta$ responses by endometrial GECs} reduces HIV-1 replication

In addition to measuring the effect of HIV-1-induced IFN $\beta$ on HIV-1 infection we also analyzed the effect of IFN $\beta$ on HIV-1 replication. To test the ability of epithelial supernatants containing IFN $\beta$ to affect infection and replication in T cells, we utilized a 1G5 HIV-1 LTR assay that has previously been used as a proxy assay for measuring HIV-1 replication. ${ }^{39}$

1G5 Jurkat $\mathrm{T}$ cells containing stably transfected HIV-LTRluciferase were activated by addition of PMA that drives HIV-1 LTR. To measure effect of epithelial supernatants containing IFN $\beta$ on HIV-1 LTR activation, supernatants from gp120 or mock-treated GEC cultures were treated with anti-IFN $\beta$ or anti-TNF $\alpha$ or isotype control antibody before addition to 1G5 cells activated by PMA. HIV-1 LTR activation was increased in 1G5 cells incubated with supernatants from gp120-treated cultures compared with mock treatment, as expected. Furthermore, HIV-1 LTR activation was significantly enhanced in the presence of anti-IFN $\beta$ antibody and reduced to background level in the presence of anti-TNF $\alpha$ antibody, whereas isotype antibody did not have any effect on HIV-1 LTR activity (Figure $7 \mathrm{~b}$ ). These results indicate that IFN $\beta$ present in epithelial cells treated with gp120 has an effect on decreasing HIV-1 LTR activation, a proxy for HIV-1 replication, in T cells.

HIV-1 exposure of GECs induces interferon stimulatory gene expression

As IFN $\beta$ produced by the GECs in response to HIV-1 was able to create an antiviral restrictive state, likely through the induction of various ISGs, we therefore next sought to determine the gene expression profiles of GECs exposed to HIV-1 by human genome ST 2.0 array. Microarray data analysis showed that GECs induced various ISGs in response to HIV-1 at $48 \mathrm{~h}$ (Table 2). Of the various ISGs reported in Table 2, ISG15 was upregulated by 2.75 -fold by microarray and when validated by real-time PCR (qPCR) it was up by 7.80 -fold in HIV-1-exposed GECs. Other ISGs were upregulated from 2.29- to 4.12-fold when compared with mock controls by microarray. Gene expression pattern observed in microarray analysis was further validated by $\mathrm{qPCR}$ and showed similar pattern as the microarray data (Table 2).

\section{DISCUSSION}

The genital epithelial cells are the first sensors and responders in the reproductive tract mucosa. ${ }^{3}$ They are the gatekeepers that determine whether immune responses will be initiated against a certain antigen and, if so, the magnitude and quality of the response. These unique abilities are conferred on the GECs, to a large extent, by the expression of a full range of pattern recognition receptors, including TLRs. ${ }^{1}$ Previous studies, including ours, have shown that activation of GECs by TLR ligands, for example, TLR3 activation by poly I:C, can induce production of both inflammatory cytokines through NF- $\kappa B$ pathways as well as IFN $\beta$ through IRF3 pathway. ${ }^{5,6}$ Our current findings demonstrate that GECs from endometrium and cervix were also activated by HIV-1 gp120 to induce IFN $\beta$, although the induction appeared to be less potent in endocervix. The IFN $\beta$ was shown to be biologically active and capable of decreasing both HIV infection and replication. Most interestingly, the IFN $\beta$ had a protective effect on the tight junction barrier and was seen to counteract the disruptive effect of TNF- $\alpha$ also induced by HIV-1 (Figure 2). The induction was dependent upon recognition of envelope glycoprotein, as no increase in IFN $\beta$ production was seen in the absence of gp120 (env $v^{-}$mutant) or following antibody mediated neutralization of gp120. We also showed that TLR2 was responsible for recognition of gp120, as only neutralization of TLR2, but not TLR4 or TLR5, both of which are also expressed on cell surface and can bind microbial glycoproteins, resulted in abrogation of IFN $\beta$ production by GECs. Finally, we demonstrated the downstream activation of ISGs that has been shown to have a broad range of antiviral effects on the mucosal surfaces.

Although a number of other studies have shown the activation of type I IFN by HIV-1 in immune cells, particularly 

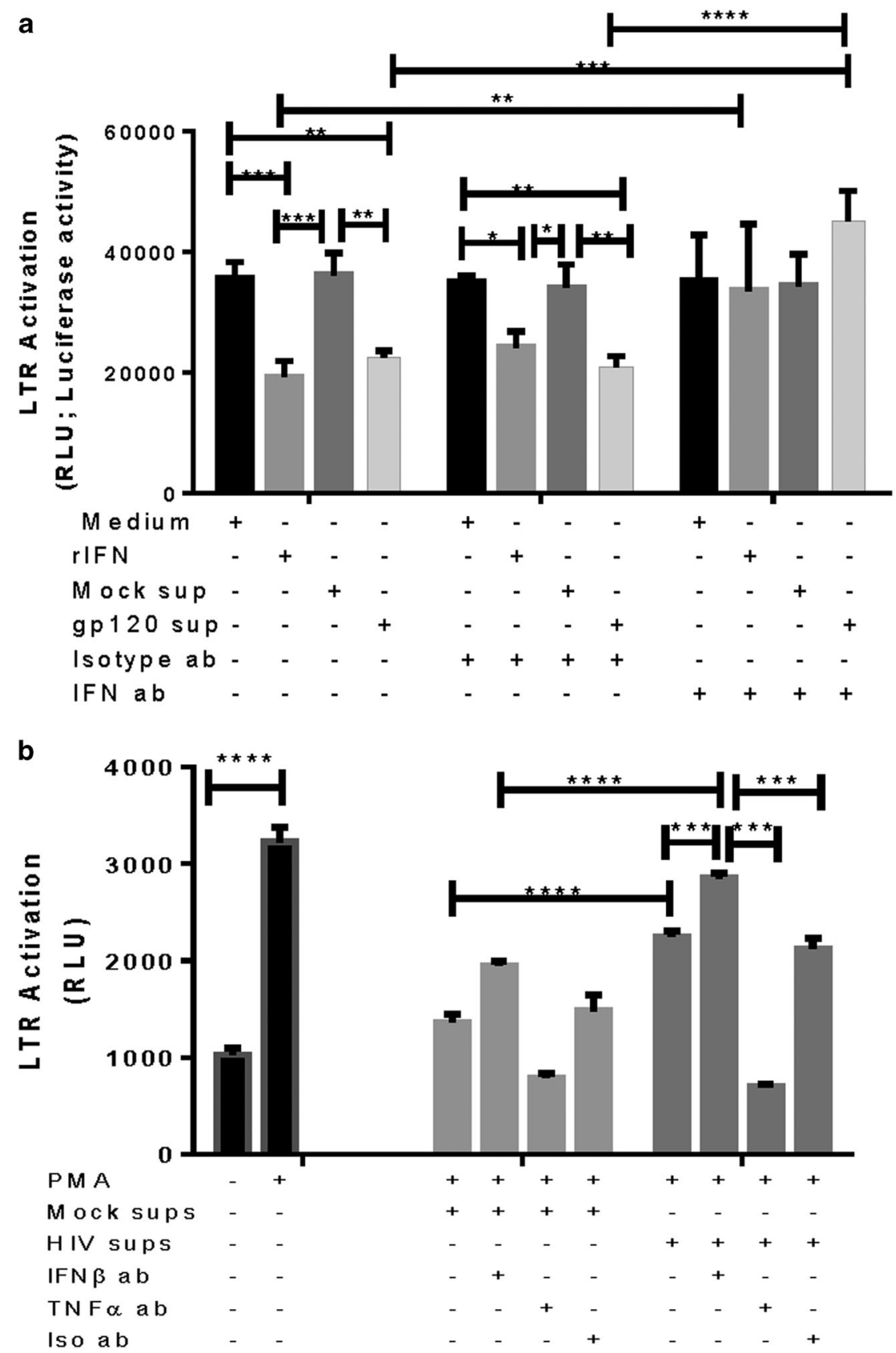

Figure 7 HIV-mediated IFN $\beta$ production in endometrial GEC supernatants decreases HIV-1 infection and HIV replication. (a) Supernatants were collected from GECs exposed to medium (mock) or gp120, added either alone or in combination with anti-IFN $\beta$ antibody or isotype antibody to TZM-bl indicator cells $1 \mathrm{~h}$ before infection with HIV-1. (b) $1 \mathrm{G} 5$ cells were activated for $24 \mathrm{~h}$ with PMA and GEC supernatants were added in the presence of either anti-IFN $\beta$ or anti-TNF $\alpha$ or isotype antibody individually. After $24 \mathrm{~h}$ of treatment, cells were lysed and luciferase activity was measured. Data are representative of three separate experiments done on three different tissues, run in duplicate. ${ }^{*} P<0.05,{ }^{* *} P<0.01,{ }^{* * *} P<0.001$ and ${ }^{* * *} P<0.0001$. GEC, genital epithelial cell; IFN $\beta$, interferon- $\beta$; PMA, phorbol 12-myristate 13-acetate; TNF $\alpha$, tumor necrosis factor- $\alpha$.

pDCs, to the best of our knowledge, this is the first report of activation of IFN $\beta$ pathway in epithelial cells by HIV-1 envelope glycoprotein., ${ }^{9,8}$ Previous studies have shown robust production of IFN $\alpha$ by pDCs in response to HIV-1. ${ }^{15}$ This interaction was shown to require two distinct interactions between the virus and cell. The first was between viral envelope and CD4 that led to endocytosis of HIV-1 and the second was the recognition of viral RNA delivered by the endosome and consequent activation of a cytoplasmic TLR pathway leading to production of IFN $\alpha$. Monocyte-derived dendritic cells, on the other hand, had poor recognition and response to HIV-1, producing low levels of IFN and failing to produce proinflammatory cytokines. ${ }^{40}$ Other immune cells including macrophages also have poor IFN responses to HIV-1 despite sensing 
Table 2 HIV-1 mediated upregulation of different interferon-stimulated genes (ISGs) in primary genital epithelial cells (GECs)

\begin{tabular}{|c|c|c|c|c|}
\hline Name & Gene & Microarray (fold changes a) & Real-time PCR (fold changes a) & Regulation \\
\hline $\mathrm{MX1}$ & $\begin{array}{l}\text { Myxovirus (influenza virus) resistance } 1 \text {, interferon-inducible protein } \\
\text { p78 }\end{array}$ & 2.60 & 5.44 & Up \\
\hline IFIT1 & Interferon-induced protein with tetratricopeptide repeats 1 & 3.82 & 8.37 & Up \\
\hline IFIT3 & Interferon-induced protein with tetratricopeptide repeats 3 & 2.59 & 2.87 & Up \\
\hline OAS1 & 2'-5'-Oligoadenylate synthetase 1 & 2.80 & 3.47 & Up \\
\hline OAS2 & 2'-5'-Oligoadenylate synthetase 1 & 4.12 & 23.82 & Up \\
\hline OAS3 & 2'-5'-Oligoadenylate synthetase 3 & 2.39 & 10.80 & Up \\
\hline BST2 & Bone marrow stromal cell antigen 2 & 2.46 & 6.90 & Up \\
\hline
\end{tabular}

aFold change values in HIV-1-exposed GECs compared with unexposed control.

HIV-1, likely because of viral immune evasion mechanisms. ${ }^{41}$ The present study shows that unlike pDCs, activation of IFN $\beta$ in GECs was mediated through cell surface TLR2 and the responses in the primary epithelial cultures were quite robust, as seen by the ability of supernatants to significantly decrease HIV-1 infection in an indicator cell line HIV-1 LTR activation in 1G5 $\mathrm{T}$ cells and viral replication in chronically HIV-1infected T cells. Similar to pDCs, the activation took place in the absence of infection, as previous studies have shown that upper GECs do not productively get infected by HIV-1. ${ }^{17}$

In addition to TLR-mediated activation of type I IFN pathway, we have also previously reported that gp120 leads to induction of proinflammatory cytokines, including TNF $\alpha$, interleukin (IL)-8, IL-6 and IL- $1 \alpha .{ }^{18} \mathrm{TNF} \alpha$, in particular, was associated with disruption of tight junctions and consequent impairment of mucosal epithelial barrier. The disruption of mucosal barriers during HIV-1 infection has been implicated in microbial translocation and immune activation. Indeed, in our studies, bacteria were observed to translocate across disrupted epithelial monolayers following exposure to HIV-1 gp120. The findings from the present study indicate that in addition to proinflammatory cytokines, the HIV envelope glycoprotein also induces IFN $\beta$, a potent innate antiviral factor. The supernatants from upper genital tract epithelial cells activated by gp120 exerted a significant inhibitory effect on both HIV-1 infection and replication in experiments conducted to specifically assess these outcomes (Figures 7), even in the presence of proinflammatory factors. Thus, the overall net outcome appears to favor an antiviral environment, as related to HIV infection and replication. This is supported by the low rates of transmission seen during heterosexual transmission showing that innate defense mechanisms in FRT under normal conditions are quite robust. However, pre-existing inflammation and/or increased number of target $\mathrm{T}$ cells could easily alter this equation. We recently combined mathematical modeling with an in vivo humanized mouse model to demonstrate that the number of target cells present in the reproductive tract are the predominant determinant in the outcome of exposure. ${ }^{42}$
Viral load was also an important criterion. Ultimately, the outcome of exposure may be decided by a number of factors in the microenvironment including number of target cells, viral load, inflammation, hormone environment and so on. Where antiviral innate immune responses fit into this equation remains to be determined.

We also examined what effect the IFN $\beta$ had on barrier functions of the epithelial cells. A number of studies have shown a mixed role for type I IFNs in HIV-1 infection, acting as a potent inhibitor for viral replication, but at the same time playing a supporting role in immune activation and pathogenesis. ${ }^{9,8}$ Our results show that IFN $\beta$ produced by GECs from the upper genital tract opposes the effects of TNF $\alpha$ and acts to protect the epithelial tight junction barrier from the disruptive effects of HIV-1 (Figure 2). A number of previous studies, including experimental and clinical ones done mostly in the context of inflammatory diseases, have shown that TNF $\alpha$ and IFN $\beta$ cross-regulate each other. ${ }^{31}$ Whether IFN $\beta$ has any direct effect on GEC tight junction barrier is currently being examined. Also, whether other proinflammatory cytokines such as IL-6 and IL- $1 \alpha$ play any role in this system and whether IFN $\beta$ modulates expression of these cytokines is not currently known. Further studies are needed to examine these complex interactions in vivo.

One surprising observation in the current study was that IFN $\beta$ was activated exclusively through TLR2, even though our previous studies show that gp120 binds to both TLR2 and TLR4 and proinflammatory cytokines are activated through both TLRs. ${ }^{19}$ Although TLR2 is known primarily for recognition of bacterial cell wall component, a number of studies have also reported that it can play a role in innate immune response to viruses. ${ }^{43,44}$ Viral glycoproteins from vaccinia, measles and gamma herpes virus have been shown to elicit TLR2-dependent type I IFN responses, primarily in cells of myeloid lineage. ${ }^{43}$ The intracellular pathways involved in TLR2-mediated induction of type I IFN are not completely clear, although it appears that endosomal localization might be involved following ligand binding. ${ }^{44}$ To the best of our knowledge this is the first report 
of TLR2-mediated activation of IFN $\beta$ in epithelial cells. Further work is needed to elucidate the detailed intracellular mechanism involved in this pathway. It is interesting to note that the overall IFN $\beta$ levels were 2-3-fold higher in the basolateral compartment of the GEC cultures compared with apical side, although there was no difference in the biological activity. As HIV-1 target cells are located primarily in the lamina propria of the genital tract underneath the epithelium, an innate IFNmediated immune response induced by GECs would be beneficial for creating an antiviral milieu in the genital tract. ${ }^{1}$ The IFN $\beta$ secreted from the basolateral side of GECs could lead to induction of ISGs in immune cells such as macrophages, DCs and T cells, making the likelihood of successful HIV-1 infection more unlikely. This inference is supported by the fact that the probability of HIV-1 transmission in the female genital tract is relatively low (1:200-1:2000 per coital act). ${ }^{2}$ Indeed, in our studies we found that IFN- $\beta$ production by GECs results in downstream signaling in the epithelial cells themselves, inducing various ISGs such as MX1, OAS1, OAS2, OAS3, ISG15 and RSAD2 as determined by DNA microarrays and confirmed by qPCR (Table 2). The ISGs reported herein has been shown to exhibit antiviral activity exerted through different mechanisms. ${ }^{45}$ Given the antiviral properties of these ISGs, it is likely that an IFN- mediated innate antiviral response mounted by GECs is beneficial in initial virus clearance as well as mucosal protection. However, the exact role of these ISGs in GECs is not known. Further in vivo studies would be needed to assess the relative contribution of GECs in mounting an effective antiviral response that decreases susceptibility to HIV-1. If this plays a significant role, it could be exploited for antiviral prophylaxis.

Although our studies provide evidence for the first time that HIV-1 gp120 can induce type I IFN pathway in upper genital epithelial cells, the in vivo significance of these studies needs to be confirmed in clinical studies. Our primary epithelial cells are isolated from endocervix and endometrial hysterectomy tissues and demonstrate the induction of IFN $\beta$ by HIV gp120 in upper genital tract epithelium. Whether this pathway is also activated in the vaginal epithelium, which is the site of primary exposure from infected semen, needs to be further examined. Whether viral or gp120 concentrations reach sufficient levels in the upper genital tract to initiate this type of response remains to be proved, although there is growing evidence that sufficient amounts of virus does reach the upper genital tract to initiate infection. ${ }^{46}$ Non-human primate studies have shown that following intravaginal inoculation with SIV, endocervix is the preferred site for initiation of infection in macaques. ${ }^{47}$ More recent studies have shown simian immunodeficiency virus (SIV) infection in ovaries and fallopian tubes of macaques, following intravaginal inoculation. ${ }^{48}$ The amount of virus present in seminal plasma has been shown to vary widely, ranging from $10^{2}$ to $10^{7}$ copies $/ \mathrm{ml}$, based on stage of infection and antiretroviral treatment, with most studies reporting a median range of 3-4 $\log 10$ copies/ml. ${ }^{49-52}$ However, based on these concentrations in semen, it is difficult to estimate per cell exposure in vivo. Finally, the evidence regarding the amount of gp120 in body fluids is controversial and remains unresolved. The assumption that gp120 should equate to viral counts is simplistic, given that gp120 can be present not only on the virions but also in soluble form secreted by infected cells. ${ }^{53}$ One study measured much higher concentration of gp120 in tissues such as lymph nodes and spleen $(>300 \mathrm{ng} / \mathrm{ml})$ in the absence of plasma viral load than previously estimated. ${ }^{54}$ However, others have estimated that the range of gp120 in serum of an HIV-1-infected individual is likely between $500 \mathrm{ng} /$ $\mathrm{ml}$ and $5 \mu \mathrm{g} / \mathrm{ml}$ when concentrations of soluble gp120, cellassociated gp120 and virion-associated gp120 are added. ${ }^{55}$ Given the above caveats, the studies summarized here demonstrate mechanistic feasibility and more evidence needs to be gathered from clinical studies to validate their in vivo relevance. A recent study showed that type I IFN-related antiviral factors (APOBEG-3G, TRIM5-a, SAMDH-1, STING, TBk1) were upregulated in the oral epithelium of exposed seronegative individuals, indicating a role for type I IFN-mediated protection against HIV-1 infection in oral mucosa. ${ }^{56}$ More clinical studies are needed to examine this association in other mucosal tracts.

In summary, we have provided novel experimental evidence that HIV-1 envelope glycoprotein is recognized by TLR2 expressed on primary human epithelial cells of the female upper genital tract, leading to activation of an innate anti-viral IFN response that is biologically relevant, acts to protect the mucosal barrier in the genital tract and can significantly decrease HIV-1 infection and replication.

\section{CONFLICT OF INTEREST}

The authors declare no conflict of interest.

\section{ACKNOWLEDGEMENTS}

We thank the Pre-Op Clinic Staff for their assistance in obtaining informed consents and Clinical Pathology Staff at Hamilton Health Sciences Centre for their assistance in providing genital tract tissues. We thank the women who donated their tissues for this study. We are also thankful for the reagents obtained from NIH AIDS Research and Reference Reagent program, Division of AIDS, NIAID, NIH: TZM-bl from Dr John C Kappes, Dr Xiaoyun Wu and Tranzyme Inc. This research was supported by grants from the Canadian Institutes of Health Research (CIHR Operating Grant FRN 126019 (to CK); CIHR Team Grant on Mucosal Immunology of HIV Vaccine Development FRN 138657 (to CK); and the Ontario HIV Treatment Network (OHTN) Applied HIV Research Chair AHRC 779 (to CK). VHF was supported by CIHR Banting Scholarship.

\section{AUTHOR CONTRIBUTIONS}

$\mathrm{AN}$ and CK conceptualized the study, designed the experiments and wrote the paper; AN, SD, MAZ, VHF and JK performed the experiments and analyzed results in Figures 1, 3, 4, 5, 6 and Supplementary Figures 2 and 3; SD and AN performed the experiments and analyzed results for Figure 2. MAZ and AN performed experiments and analyzed results in Figure 7 and Supplementary Figure 1. MWW worked on revisions to the paper. MO and MJT constructed HIV-1 env deleted clone and provided technical assistance. AAA purified and provided FimH for Figure 4. 
DMEB provided plasmids and technical assistance for transfection experiments in Figure 5. CK coordinated and supervised the study. All authors reviewed the results and approved the final version of the manuscript.

1 Ferreira VH, Kafka JK, Kaushic C. Influence of common mucosal cofactors on HIV infection in the female genital tract. Am J Reprod Immunol 2014; 71: 543-554.

2 Shattock R, Moore J. Inhibiting sexual transmission of HIV-1 infection. Nat Rev Microbiol 2003; 1: 25-34.

3 Kaushic C. HIV-1 infection in the female reproductive tract: role of interactions between HIV-1 and genital epithelial cells. Am J Reprod Immunol 2011; 65: 253-260.

4 Wira CR, Fahey JV, Sentman CL, Pioli PA, Shen L. Innate and adaptive immunity in female genital tract: cellular responses and interactions. Immunol Rev 2005; 206: 306-335.

5 Nazli A, Yao X-D, Smieja M, Rosenthal KL, Ashkar AA, Kaushic C. Differential induction of innate anti-viral responses by TLR ligands against Herpes simplex virus, type 2, infection in primary genital epithelium of women. Antiviral Res 2009; 81: 103-112.

6 Schaefer TM, Fahey JV, Wright JA, Wira CR. Innate immunity in the human female reproductive tract: antiviral response of uterine epithelial cells to the TLR3 agonist poly(I:C). J Immunol 2005; 174: 992-1002.

7 Fichorova RN, Anderson DJ. Differential expression of immunobiological mediators by immortalized human cervical and vaginal epithelial cells. Biol Reprod 1999; 60: 508-514.

8 Bosinger SE, Utay NS. Type I interferon: understanding its role in HIV pathogenesis and therapy. Curr HIV/AIDS Rep 2015; 12: 41-53.

9 Sivro A, Su RC, Plummer FA, Ball TB. Interferon responses in HIV infection: from protection to disease. AIDS Rev 2014; 16: 43-51.

10 Meylan PR, Guatelli JC, Munis JR, Richman DD, Kornbluth RS. Mechanisms for the inhibition of HIV replication by interferons-alpha, -beta, and -gamma in primary human macrophages. Virol 1993; 193: 138-148.

11 Der SD, Zhou A, Williams BR, Silverman RH. Identification of genes differentially regulated by interferon alpha, beta, or gamma using oligonucleotide arrays. Proc Natl Acad Sci 1998; 95: $15623-15628$.

12 de Veer MJ, Holko M, Frevel M, Walker E, Der S, Paranjape JM et al. Functional classification of interferon-stimulated genes identified using microarrays. J Leukoc Biol 2001; 69: 912-920.

13 Schoggins JW, Wilson SJ, Panis M, Murphy MY, Jones CT, Bieniasz P et al. A diverse range of gene products are effectors of the type I interferon antiviral response. Nature 2011; 472: 481-485.

14 Nasr N, Maddocks S, Turville SG, Harman AN, Woolger N, Helbig KJ et al. HIV-1 infection of human macrophages directly induces viperin which inhibits viral production. Blood 2012; 120: 778-788.

15 Fonteneau JF, Larsson M, Beignon AS, McKenna K, Dasilva I, Amara A et al. Human immunodeficiency virus type 1 activates plasmacytoid dendritic cells and concomitantly induces the bystander maturation of myeloid dendritic cells. J Virol 2004; 78: 5223-5232.

16 Machmach K, Leal M, Gras C, Viciana P, Genebat M, Franco E et al. Plasmacytoid dendritic cells reduce HIV production in elite controllers. J Virol 2012; 86: 4245-4252.

17 Ferreira VH, Dizzell S, Nazli A, Kafka JK, Mueller K, Nguyen PV et al. Medroxyprogesterone acetate regulates HIV-1 uptake and transcytosis but not replication in primary genital epithelial cells, resulting in enhanced T-cell infection. J Inf Dis 2015; 211: 1745-1756.

18 Nazli A, Chan O, Dobson-Belaire WN, Ouellet M, Tremblay MJ, GrayOwen SD et al. Exposure to HIV-1 directly impairs mucosal epithelial barrier integrity allowing microbial translocation. PLoS Pathog 2010; 6: e1000852.

19 Nazli A, Kafka JK, Ferreira VH, Anipindi V, Mueller K, Osborne BJ et al. HIV-1 gp120 induces TLR2- and TLR4 mediated innate immune activation in human female genital epithelium. J Immunol 2013; 191: 4246-4258.

20 MacDonald EM, Savoy A, Gillgrass A, Fernandez S, Smieja M, Rosenthal $\mathrm{KL}$ et al. Susceptibility of human female primary genital epithelial cells to herpes simplex virus, type- 2 and the effect of TLR3 ligand and sex hormones on infection. Biol Reprod 2007; 77: 1049-1059.
21 Kaushic C, Nazli A, Ferreira VH, Kafka JK. Primary human epithelial cell culture system for studying interactions between female upper genital tract and sexually transmitted viruses, HSV-2 and HIV-1. Methods 2011; 55: 114-121.

22 Takeuchi Y, McClure MO, Pizzato M. Identification of gammaretroviruses constitutively released from cell lines used for human immunodeficiency virus research. J Virol 2008; 82: 12585-12588.

23 Wei X, Decker JM, Liu H, Zhang Z, Arani RB, Kilby JM et al. Emergence of resistant human immunodeficiency virus type 1 in patients receiving fusion inhibitor (T-20) monotherapy. Antimicrob Agents Chemother 2002; 46: 1896-1905.

24 Derdeyn CA, Decker JM, Sfakianos JN, Wu X, O'Brien WA, Ratner L et al. Sensitivity of human immunodeficiency virus type 1 to the fusion inhibitor T-20 is modulated by coreceptor specificity defined by the V3 loop of gp120. J Virol 2000; 74: 8358-8367.

25 Platt EJ, Wehrly K, Kuhmann SE, Chesebro B, Kabat D. Effects of CCR5 and CD4 cell surface concentrations on infections by macrophagetropic isolates of human immunodeficiency virus type 1 . J Virol 1998; 72: 2855-2864.

26 Nakhaei P, Mesplede T, Solis M, Sun Q, Zhao T, Yang L et al. The E3 ubiquitin ligase Triad3A negatively regulates the RIG-I/MAVS signaling pathway by targeting TRAF3 for degradation. PLoS Pathog 2009; 5: e1000650.

27 Ferreira VH, Nazli A, Dizzell SE, Mueller K, Kaushic C. The antiinflammatory activity of curcumin protects the genital mucosal epithelial barrier from disruption and blocks replication of HIV-1 and HSV-2. PLOS ONE 2015; 1: e0124903.

28 Ott DE, Chertova EN, Busch LK, Coren LV, Gagliardi TD, Johnson DG. Mutational analysis of the hydrophobic tail of the human immunodeficiency virus type $1 \mathrm{p} 6$ (Gag) protein produces a mutant that fails to package its envelope protein. J Virol 1999; 73: 19-28.

29 Meager A. Biological assays for interferons. J Immunol Methods 2002; 261: 21-36.

30 Clark K, Plater L, Peggie M, Cohen P. Use of the pharmacological inhibitor BX795 to study the regulation and physiological roles of TBK1 and IkappaB kinase epsilon: a distinct upstream kinase mediates Ser-172 phosphorylation and activation. J Biol Chem 2009; 284: 14136-14146.

31 Cantaert T, Baeten D, Tak PP, van Baarsen LG. Type I IFN and TNFalpha cross-regulation in immune-mediated inflammatory disease: basic concepts and clinical relevance. Arthritis Res Ther 2010; 12: 219-229.

32 Wang J, Basagoudanavar SH, Wang X, Hopewell E, Albrecht R, Garcia-Sastre A et al. NF-kappa B RelA subunit is crucial for early IFN-beta expression and resistance to RNA virus replication. J Immunol 2010; 185: 1720-1729.

33 Kawai T, Akira S. TLR signaling. Semin Immunol 2007; 19: 24-32.

34 Dietrich N, Lienenklaus S, Weiss S, Gekara NO. Murine Toll-like receptor 2 activation induces type I interferon responses from endolysosomal compartments. PLOS ONE 2010; 5: e10250.

35 Mossman KL, Mian MF, Lauzon NM, Gyles CL, Lichty B, Mackenzie R et al. Cutting edge: FimH adhesin of type 1 fimbriae is a novel TLR4 ligand. J Immunol 2008; 181: 6702-6706.

36 Asea A, Rehli M, Kabingu E, Boch JA, Bare O, Auron PE et al. Novel signal transduction pathway utilized by extracellular HSP70: role of Toll-like receptor (TLR) 2 and TLR4. J Biol Chem 2002; 277. 15028-15034.

37 Jurk M, Heil F, Vollmer J, Schetter C, Krieg AM, Wagner $\mathrm{H}$ et al. Human TLR7 or TLR8 independently confer responsiveness to the antiviral compound R-848. Nat Immunol 2002; 3: 499.

38 Park BS, Lee J-O. Recognition of lipopolysaccharide pattern by TLR4 complexes. Exp Mol Med 2013; 45: e66.

39 Ferreira VH, Nazli A, Khan G, Firoz Mian M, Ashkar AA, Gray-Owen S et al. Endometrial epithelial cell responses to co-infecting viral and bacterial pathogens in the genital tract can activate the HIV-1 LTR in an NFкB and AP-1 dependent manner. J Infect Dis 2011; 204: 299-308.

40 Granelli-Piperno A, Golebiowska A, Trumpfheller C, Siegal FP, Steinman RM. HIV-1-infected monocyte-derived dendritic cells do not undergo maturation but can elicit IL-10 production and $\mathrm{T}$ cell regulation. PNAS 2004; 101: 7669-7674.

41 Marsili G, Remoli AL, Sgarbanti M, Perrotti E, Fragale A, Battistini A. HIV-1, interferon and the interferon regulatory factor system: an interplay between induction, antiviral responses and viral evasion. Cytokine Growth Factor Rev 2012; 23: 255-270. 
42 Nguyen PV, Wessels JM, Mueller K, Vahedi F, Anipindi V, Verschoor CP et al. Frequency of human CD45+ target cells is a key determinant of intravaginal HIV-1 infection in humanized mice. Sci Rep 2017; 7: 15263-15278.

43 Stack J, Doyle SL, Connolly DJ, Reinert LS, O'Keeffe KM, McLoughlin RM et al. TRAM is required for TLR2 endosomal signaling to type I IFN induction. J Immunol 2014; 193: 6090-6102.

44 Barbalat R, Lau L, Locksley RM, Barton GM. Toll-like receptor 2 on inflammatory monocytes induces type I interferon in response to viral but not bacterial ligands. Nat Immunol 2009; 10: 1200-1207.

45 Sadler AJ, Williams BR. Interferon-inducible antiviral effectors. Nat Rev Immunol 2008; 8: 559-568.

46 Haase AT. Targeting early infection to prevent HIV-1 mucosal transmission. Nature 2010; 464: 217-223.

47 Li Q, Estes JD, Schlievert PM, Duan L, Brosnahan AJ, Southern PJ et al. Glycerol monolaurate prevents mucosal SIV transmission. Nature 2009; 458: 1034-1038.

48 Stieh DJ, Maric D, Kelley ZL, Anderson MR, Hattaway HZ, Beilfuss BA et al. Vaginal challenge with an SIV-based dual reporter system reveals that infection can occur throughout the upper and lower female reproductive tract. PLoS Pathog 2014; 10: e1004440.

49 Gupta P, Mellors J, Kingsley L, Riddler S, Singh MK, Schreiber S et al. High viral load in semen of human immunodeficiency virus type 1 -infected men at all stages of disease and its reduction by therapy with protease and nonnucleoside reverse transcriptase inhibitors. $J$ Virol 1997; 71: 6271-6275.

50 Stekler J, Sycks BJ, Holte S, Maenza J, Stevens CE, Dragavon J et al. HIV dynamics in seminal plasma during primary HIV infection. AIDS Res Hum Retroviruses. 2008; 24: 1269-1274.

51 Hoffman JC, Anton PA, Baldwin GC, Elliott J, Anisman-Posner D, Tanner $\mathrm{K}$ et al. Seminal plasma HIV-1 RNA concentration is strongly associated with altered levels of seminal plasma interferon-gamma, interleukin-17, and interleukin-5. AIDS Res Hum Retroviruses 2014; 30: 1082-1088.
52 Tachet A, Dulioust E, Salmon D, De Almeida M, Rivalland S, Finkielsztejn $L$ et al. Detection and quantification of HIV-1 in semen: identification of a subpopulation of men at high potential risk of viral sexual transmission. AIDS 1999; 13: 823-831.

53 Klasse PJ, Moore JP. Is there enough gp120 in the body fluids of HIV-1-infected individuals to have biologically significant effects? Virology 2004; 323: 1-8.

54 Santosuosso M, Righi E, Lindstrom V, Leblanc PR, Poznansky MC. HIV-1 envelope protein gp120 is present at high concentrations in secondary lymphoid organs of individuals with chronic HIV-1 infection. J Infect Dis 2009; 200: 1050-1053.

55 Cummins NW, Rizza SA, Badley AD. How much gp120 is there? J Infect Dis 2010; 201: 1273-1274 author reply 4-5.

56 Cervantes CA, Oliveira LM, Manfrere KC, Lima JF, Pereira NZ, Duarte AJ et al. Antiviral factors and type I/III interferon expression associated with regulatory factors in the oral epithelial cells from HIV-1-serodiscordant couples. Sci Rep 2016; 6: 25875.

(c) (i) $(-)$ This work is licensed under a Creative Commons Attribution-NonCommercial-NoDerivs $\quad \mathbf{4 . 0}$

International License. The images or other third party material in this article are included in the article's Creative Commons license, unless indicated otherwise in the credit line; if the material is not included under the Creative Commons license, users will need to obtain permission from the license holder to reproduce the material. To view a copy of this license, visit http://creativecommons.org/licenses/by-nc-nd/4.0/

(C) The Author(s) 2018

Supplementary Information for this article can be found on the Cellular \& Molecular Immunology website (http://www.nature.com/ $\mathrm{cmi}$ ) 